\title{
The early gene Broad is involved in the ecdysteroid hierarchy governing vitellogenesis of the mosquito Aedes aegypti
}

\author{
L Chen, J Zhu, G Sun and A S Raikhel
}

Department of Entomology and Institute for Integrative Genome Biology, University of California, Riverside, California 92521, USA

(Requests for offprints should be addressed to A S Raikhel; Email: alexander.raikhel@ucr.edu)

\begin{abstract}
The broad (br) gene, encoding a family of $\mathrm{C} 2 \mathrm{H} 2$ type zinc-finger DNA-binding proteins, has been shown to act as a crucial member of the 20-hydroxyecdysone (20E) regulatory hierarchy in the fruitfly, Drosophila melanogaster and the moth, Manduca sexta. In this study, we have shown that the br gene is involved in the 20E-regulatory hierarchy controlling vitellogenesis in the mosquito, Aedes aegypti. Unlike $E 74$ and $E 75$ early genes, expression of $b r$ was activated in previtellogenic females, during a juvenile hormone $(\mathrm{JH})$-dependent period. The levels of $\mathrm{Z1}, \mathrm{Z2}$ and $\mathrm{Z4}$ isoform mRNA were elevated in the fat body of 2-day-old females after in vitro exposure to JH III. However, JH III repressed 20E activation of br in 3to 5-day-old females, indicating a switch in hormonal commitment. Expression of Z1, Z2 and Z4 was stimulated after blood feeding in both vitellogenic tissues, the fat body and the ovary, corresponding to peaks of ecdysteroid titers. In the fat body, the mRNA profiles of these three isoforms correlated well with those of yolk protein precursor (YPP) genes. These BR isoforms were activated by 20E in fat bodies cultured in vitro and behaved as early genes, with a self-repressive autoregulatory loop that can be blocked by the protein inhibitor, cyclohexamide. Multiple binding sites for all four BR isoforms were present in the $5^{\prime}$-regulatory region of the major YPP gene, vitellogenin $(V g)$. Effects of BR isoforms on the expression of $V g$ have been demonstrated by cell transfection analysis. In particular, BR isoforms by themselves had no effects on the $\mathrm{Vg}$ promoter. However, Z1 and Z4 each repressed Aedes aegypti ecdysone receptor $(\mathrm{EcR}) /$ Ultraspiracle (USP)-mediated 20E activation of the $\mathrm{Vg}$ promoter, while Z2 enhanced activation of the $V g$ promoter by AaEcR/AaUSP in the presence of $20 \mathrm{E}$. Z3 had no obvious effect in the same experiment. These results suggested that BR isoforms are essential for proper activation and termination of the $V g$ gene in response to 20E. Overall, our study implicated $b r$ in the regulation of mosquito vitellogenesis.
\end{abstract}

Journal of Molecular Endocrinology (2004) 33, 743-761

\section{Introduction}

In anautogenous mosquitoes, egg maturation requires a blood meal. As a consequence, mosquitoes are vectors of numerous devastating human diseases. Vitellogenesis is the key process in egg maturation, which involves production of enormous amounts of yolk protein precursors (YPPs) by the female fat body, an insect metabolic tissue. This process is mainly governed by two insect hormones: juvenile hormone (JH) III and 20-hydroxyecdysone (20E). Induction of the syn- thesis of yolk protein precursors in the fat body requires 20E, while JH III prepares newly emerged mosquitoes to accommodate the intense physiological demands of vitellogenesis, and to become competent to respond to signals associated with blood feeding, including 20E (Flanagan \& Hagedorn 1977, Shapiro \& Hagedorn 1982, Hagedorn 1985). In keeping with the majority practice among researchers in the field, we use ecdysteroid as the generic term for steroidal insect molting hormones, reserving the term ecdysone for the specific chemical compound $2 \beta, 3 \beta, 14 \alpha$, 
22R,25-pentahydroxy-5 $\beta$-cholest-7-en-6-one, originally known as $\alpha$-ecdysone. The abbreviation $20 \mathrm{E}$ is used to refer to 20-hydroxyecdysone $(2 \beta, 3 \beta, 14 \alpha, 20 \mathrm{R}, 22 \mathrm{R}, 25$-pentahydroxy-5 $\beta$-cholest7 -en-6-one), the highly active ecdysone metabolite believed to serve as the active hormone in most well-characterized responses.

Vitellogenesis is initiated only after a female mosquito ingests vertebrate blood (Hagedorn 1989, Raikhel 1992, Dhadialla \& Raikhel 1994). The massive yolk protein synthesis peaks around $24 \mathrm{~h}$ post blood meal (24 h PBM), then declines, terminating by $30-36 \mathrm{~h}$ PBM. Blood feeding triggers a hormonal cascade, leading to the elevation of $20 \mathrm{E}$, which is secreted by activated ovaries, under the control of egg development neurosecretory hormone (Shapiro \& Hagedorn 1982). The changing profile of 20E titers in female mosquitoes is tightly correlated with the expression of YPP genes, rising with yolk protein production and falling when vitellogenesis ceases (Hagedorn 1983, 1985, Raikhel 1992, Dhadialla \& Raikhel 1994). The genes encoding three YPPs - vitellogenin $(\mathrm{Vg})$, vitellogenic carboxypeptidase (VCP) and vitellogenic cathepsin $\mathrm{B}(\mathrm{VCB})$ - are activated in fat bodies cultured in vitro upon addition of physiological doses of $20 \mathrm{E}$, which suggests that these three genes are regulated by this hormone (Cho \& Raikhel 1992, Cho et al. 1999, Deitsch et al. 1995).

The molecular mechanism of $20 \mathrm{E}$ action has been dissected in detail during Drosophila and Manduca metamorphosis. In insects, the steroid hormone $20 \mathrm{E}$ functions by signaling through a heterodimer of nuclear receptors, consisting of the ecdysone receptor (EcR) and Ultraspiracle (USP). USP is an insect homologue of the mammalian retinoid X receptor (Yao et al. 1992). EcR/USP acts as a ligand-dependent transcription factor which regulates the activity of hormone responsive early genes, including E74, E75 and broad (br). In turn, the expression of those early genes is necessary for the induction of a large number of late genes and repression of the early genes themselves (Ashburner 1972, Burtis et al. 1990, Segraves \& Hogness 1990, Cherbas et al. 1991, DiBello et al. 1991, Guay \& Guild 1991, Koelle et al. 1991, Yao et al. 1992, 1993, Fletcher \& Thummel 1995b).

The $b r$ gene has been identified as a key gene required for insect molting, metamorphosis and oogenesis. In both Drosophila and Manduca, br induction only occurs in the final larval instar. BR protein activates the pupal program and suppresses both the larval and adult programs. JH application at the onset of the adult molt causes re-expression of $b r$ and the formation of a second pupal cuticle, suggesting that BR is sufficient to mediate the status quo action of JH (Zhou \& Riddiford 2002). Similarly, RNAi knock-down of $b r$ in the silkmoth Bombyx mori results in the failure of animals to complete the larval-pupal transition or in later morphogenetic defects (Uhlirova et al. 2003). The br gene is expressed in a dynamic pattern during oogenesis. It is activated by ecdysteroid in follicle cells at stage 6 of oogenesis, where it is essential for the control of endoreplication and chorion gene amplification. Later, $b r$ is also involved in cell migration, as well as morphogenesis of chorionic appendages (Tzolovsky et al. 1999).

$\mathrm{Br}$ encodes a family of four classes of protein isoforms (Z1-Z4), which share a common aminoterminal core domain, alternatively spliced to four distinct carboxy-terminal domains bearing pairs of zinc-finger DNA-binding domains (DiBello et al. 1991, Bayer et al. 1996). The common core region contains a highly conserved 120 amino acids, called the BTB or POZ domain; this appears to be involved in protein-protein interaction (Bardwell \& Treisman 1994, Zollman et al. 1994). The $b r$ gene is defined by mutations that comprise three classical complementation groups, br, reduced bristles on palpus $(r b p)$ and $2 B c$, as well as a non-complementing nonpupariating (npr) class (Kiss et al. 1988). The BR isoforms are critical mediators of the ecdysteroid hierarchy because they are required in the regulation of intermolt, early and late gene activities in Drosophila (Belyaeva et al. 1980, Karim et al. 1993). The npr mutant lacks all of the functional BR isoforms, and larvae are viable and initiate wandering behavior at the end of the third instar, but die without pupariating (Stewart et al. 1972, Kiss et al. 1978). Alleles that belong to the $b r$, $r b p$ and $2 B c$ complementing functions result in lethality somewhat later in development, with severe morphological defects (Belyaeva et al. 1980, Kiss et al. 1988, Restifo \& Merrill 1994). Mutations that reduce or eliminate $b r$ function disrupt the expression of all classes of 20E-responsive genes (Belyaeva et al. 1981, Lepesant et al. 1986, Guay \& Guild 1991, Nelson et al. 1991, Karim et al. 1993). 
The ecdysteroid-triggered regulatory hierarchy during mosquito vitellogenesis is reminiscent of the one utilized in Drosophila metamorphosis. However, the individual participants in the hierarchy are uniquely utilized to respond to the biological needs of the reproducing insects (Zhu et al. 2003a,b). DNA-binding sites of EcR/USP, E74 and E75 have all been identified in the 5 '-regulatory region of the $V g$ gene (Kokoza et al. 2001). Both genetic studies of the $V g$ gene utilizing Aedes transformation and cell transfection assays have indicated that EcR/USP is required for initiation of stage-specific, 20E-mediated, $V g$ gene expression (Martin et al. 2001). Moreover, chromatin immunoprecipitation assays have demonstrated that the $V g$ promoter was loaded with EcR/USP heterodimer in vivo shortly after blood feeding (Zhu et al. 2003a). E74 and E75 are induced by the elevation of $20 \mathrm{E}$ titers after blood feeding in the fat body, and their protein products are essential for the high level expression of the $V g$ gene in response to $20 \mathrm{E}$ during vitellogenesis (Pierceall et al. 1999, Kokoza et al. 2001, Sun et al. 2002, 2004). Therefore, the Vg gene is the target of direct and indirect regulation by 20E (Raikhel et al. 2003).

Sequence analysis of the $A$. aegypti $V g$ regulatory region also revealed the presence of putative binding sites of $\mathrm{BR}$, prompting us to isolate and characterize the mosquito homologue of $\mathrm{BR}$. Here, we report the cloning of four mosquito BR isoforms (Aedes aegypti (Aa) BR Z1-Z4). Transcripts of $A a B R$ increase in the fat body and ovary following the blood meal. In vitro culture experiments demonstrated that $b r$ expression was induced by $20 \mathrm{E}$ in the fat body. In cell transfection assays, AaBR isoforms showed distinct AaEcR/AaUSPmediated effects on the $V g$ promoter activity: AaBR $\mathrm{Z} 2$ as an activator and AaBR Z1 and AaBR Z4 as repressors. These findings suggest that $A a B R$ is involved in mediating the $20 \mathrm{E}$ response in the fat body and ovaries during mosquito vitellogenesis.

\section{Materials and Methods}

\section{Animals}

Mosquitoes, $A$. aegypti, were raised as described by Hays \& Raikhel (1990). Larvae were fed a standard diet (Lea 1964) and adults were fed on 10\% sucrose solution continuously by wick. Adult females, 3-5 days after eclosion, were fed on anesthetized white rats to initiate vitellogenesis. All dissections were performed in Aedes physiological saline (Hagedorn et al. 1977) at room temperature.

\section{RNA extraction, RT and real-time PCR}

Dissected fat bodies were homogenized with a motor-driven pellet pestle mixer (Kontes, Vineland, New Jersey, USA) and lysed by Trizol reagent (Invitrogen). RNA was isolated following the manufacturer's protocol. Contaminating genomic DNA was removed by treatment with RNase-free DNase I (Invitrogen). RT was carried out using an Omniscript reverse transcriptase kit (Qiagen) in a $20 \mu \mathrm{l}$ reaction mixture, containing random primers and $1 \mu \mathrm{g}$ total RNA at $37^{\circ} \mathrm{C}$ for $1 \mathrm{~h}$. Two microliters of cDNA from this reaction were subjected to PGR reaction by using $A a B R$ isoform-specific primers $\left(30\right.$ cycles of $95^{\circ} \mathrm{C}$ for $30 \mathrm{~s}$, $56^{\circ} \mathrm{C}$ for $30 \mathrm{~s}, 68^{\circ} \mathrm{C}$ for $\left.60 \mathrm{~s}\right)$. PCR products were separated on a $1 \%$ agarose gel and confirmed by Southern analysis.

Real-time PCR was performed using the iCycler iQ system (Bio-Rad, Hercules, California, USA), as previously reported (Zhu et al. 2003b). Reactions were performed in 96-well plates with a QuantiTect SYBR PGR kit (Qiagen). In order to quantify relative gene expression, standard curves were generated, using 10-fold serial dilutions of cDNA pools containing high concentrations of the gene of interest. The protocol used for amplifying the cDNA product was 40 cycles of $95^{\circ} \mathrm{C}$ for $30 \mathrm{~s}$, $59{ }^{\circ} \mathrm{C}$ for $45 \mathrm{~s}$, followed by melting curve analysis to detect specific product amplification. Samples from three individual organ culture experiments were used for real-time PCR. Each sample was analyzed in triplicate and normalized to the internal control, $\beta$-actin mRNA. Real-time data were collected by the Icycler IQ Real Time Detection System Software V.3.0 for Windows. Raw data were exported to Excel (Microsoft, Seattle, WN, USA) for analysis. Real-time PCR primers: Z1 forward primer: TTGAAAAGGTAGATATGAGGGG; Z1 reverse primer: CGGCTTCATGTTTCGATGAT AG; Z2 forward primer: CGACATATGGGTGA TAAACACGC; Z2 reverse primer: CCTGGACG GGACTTATGATACG; Z3 forward primer: GCGGCAAGAATGTCGCTACTGTC; Z3 reverse primer: TATACGGGGGGTGGGAGAAT 
TC; Z4 forward primer: TTAAACGACTCGA GCGGGGG; Z4 reverse primer: GGTTGCAGG TGGTGTTGGTG.

\section{Cloning and sequencing of cDNAs}

Drosophila BR shares significant sequence similarity with its counterparts in Manduca at BTB domains and C-terminal zinc-finger regions (Zhou et al. 1998). This allowed us to isolate mosquito homologues of BR by utilizing a PCR-based strategy. First, a pair of degenerate primers $(\mathrm{Pd})$ was designed based on highly conserved regions in the BTB domain of BR: Pd forward, 5'-SIGGIAA RCAYTAYGGICARTTYACITG-3'; Pd reverse, 5'-CKICAITAITGICAYTGRTTICKRTGRTG-3' (Zollman et al. 1994). RT-PCR with the fat body total RNA from the female adult at 3-24 h PBM yielded a single $260 \mathrm{bp}$ product, the putative amino acid sequence of which shared 97\% identity with BTB domains of Drosophila BR and 100\% identity with BTB domain of Anopheles gambiae (Fig. 1A). The sequence of BR BTB domain of Anopheles gambiae was obtained from the National Center for Biotechnology Information (NCBI). Next, degenerate primers corresponding to the isoform-specific zinc-finger regions were designed based on alignment of the C-terminal regions of BR from Drosophila and Manduca (Bayer et al. 1996, Zhou et al. 1998). These were then used in RT-PCR, in combination with a primer derived from the BTB-domain coding sequence. Four $1.6 \mathrm{~kb}$ different fragments were obtained, and DNA sequence analyses predicted that they encoded four different $\mathrm{C} 2 \mathrm{H} 2$ zinc-finger domains homologous to those of Z1 (96\%), Z2 (96\%), Z3 (100\%) and Z4 (98\%) of Drosophila (Fig. 1B). Those sequences also showed high percentage identity to the zinc-finger domains of BR from Anopheles gambiae and Manduca (Fig. 1B). After that, the $5^{\prime}$ and $3^{\prime}$ ends of individual cDNA clones were determined, using $5^{\prime}$ - and $3^{\prime}$-rapid amplification of cDNA ends PCR. The 5'- and $3^{\prime}$-untranslated regions are isoform specific. Finally, cDNA fragments containing the entire coding regions of mosquito $\mathrm{BR}$ isoforms were obtained by RT-PCR. The sequence data of four mosquito BR isoforms have been submitted to the DDBJ/ EMBL/GenBank database under accession numbers AY499537-AY499540.

DNA sequences were analyzed by the SeqWeb (San Diego, California, USA) and BLAST NCBI,
NIH, Bethesda, MD, USA). AaBR sequence alignments were performed using the 'Multiple Sequence Alignments' program from the website, http://www.justbio.com.

\section{Electrophoretic mobility shift assays (EMSA)}

EMSAs were carried out as described previously (Wang et al. 1998). Drosophila BR consensus binding sequences were used to determine the DNAbinding activities of the AaBR isoforms. The consensus sequences that we used were TAATAAAGAAAA (Z1), TTTATCATTT (Z2), TAAAGTAAA (Z3) and ATAAACA (Z4) (von Kalm et al. 1994). Complementary oligonucleotides were annealed together, and end-labeled by $\mathrm{T} 4$ polynucleotide kinase using $\left[\gamma_{-}{ }^{32} \mathrm{P}\right]$ ATP (Perkin Elmer, Boston, Massachusetts, USA). The AaBR proteins were synthesized in vitro with the TNT system (Promega). The gel shift binding buffer $(5 \times)$ was purchased from Promega.

\section{In vitro fat body culture}

20-E and JH III (Sigma) were dissolved in ethanol and acetone respectively. A medium containing $\mathrm{JH}$ III was prepared as described by Riddiford et al. (1979), and containers and culture plates were coated with Sigmacote (Sigma). Abdominal walls with adhering fat bodies (hereafter referred to as fat bodies), which were from previtellogenic female mosquitoes (2 days and 4 days after eclosion), were incubated in an organ culture system as previously described (Deitsch et al. 1995). The dissected fat bodies were treated with $20 \mathrm{E}\left(2 \times 10^{-6} \mathrm{M}\right.$ in ethanol $)$, JH III $\left(1 \times 10^{-6} \mathrm{M}\right.$ in acetone $)$, and cycloheximide (Calbiochem, San Diego, California, USA; $1 \times 10^{-5} \mathrm{M}$ in water) (a protein syntheses inhibitor) for 4-16 h, and three groups of nine fat bodies were collected at 4-h intervals. As a control, fat bodies were incubated with $0 \cdot 1 \%$ ethanol or acetone in the culture medium.

\section{Cell culture and transient transfection assay in the Drosophila L57-3-11 cell line}

The coding region sequences of the four $\mathrm{BR}$ isoforms were inserted into the pAc5.1/V5/HisA (Invitrogen) vector and fused to a V5 and a His tag. $\operatorname{Vg} 2 \cdot 1 \mathrm{~kb}$ promoter was inserted into pGL3/firefly luciferase vector (Promega) to form the reporter 
A Aa MVD--OHFCLRWNYYOSITSAFE RL RD DEDFVDVTLACDGRSLKAHRVUL SA CSPYFR

Ag MVD--QHFCLRWNWYQSI TS AFENL RD DE DFVDVTLA.CDGRSLKAHRVVL SACSTYFR

DI MDD--QHFCLRWNWY QSSI TSAFENL RD DEAFVDVTLACEGRS IKAHRVUL SACSFYFR

MS VESQTQHFCLRWNWYQRSITSAFENL RDDEDFVDVTL ACDGKSLKAHRVUL SACSPYFR

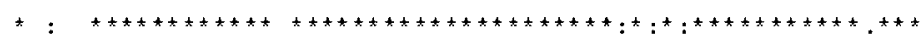

Aa ELLKS TPCKHPVIVL QDVA FTDLHALVEF IYHGEVNHORSLSSFLKTA EVLRVSGLT

Ag ELLKSTPCKHPVIVL QDVAFTDLHALVEFI YHGEVNVHQRSLS SFLKTAEILRVSGLT

DM ELLKS TPCKHPVILL QDVNFMD LHALVE I YHGEVWVHQKSLQSF LKTAEVLRVSGLT

MS ELLKS TPCKHPVIVL QDVA FTDLHALVEF I YHGEVNVHQHSLS SF FKTA EVLRVSGLT

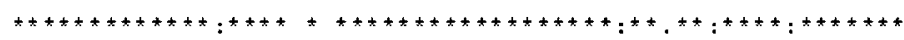

B AAZ1 FRCD FCNKUL SSLTRL KRH IQNYHURP TKEP IC IC KRVYS SLN SL RNHKS I YHRN AgZ1 FRCD PCNKNL SSLT FL RRH IONVHMRE TKEPVCNIC KRVYSSLNSL RNHKS I YHRN DMZ 1 F RCN PC NK WL SSLT TL KRH IONYHMRE TKERVC IC KRVYSSLNSL RNHKS I YHRN

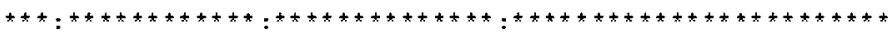

$\therefore a 22$

$\operatorname{sgz} 2$

$\operatorname{Drz2}$

$M=22$

Aaz3

Agz3

Driz3

$10 \leq 23$

$\operatorname{Aaz} 4$

$\operatorname{Dm} 24$

$M=Z 4$

C

$\operatorname{DmZ1}$

$\mathrm{AaZI}$

F SCQLC GKYL CS KS.SL KRH I. DKH. ERQE EYRC I IC ERVY CS RN SL MTH I YT THKS F SCQLC GKYL CS KA SL KRH IA DKHA.ERQE EYRC I IC ERVY CS RN SL IMTH I YTYHKS F SCQLC GKLL CS KS SL KRH IA DKHAVRQE EYRC A ICERVYCSRN SL MTH I YTYHKS F SCSLC GKYL CS KS SL KRH IA DKHA ERQE EYRC TIC ERVYCSRNSLMTH I YTYHKS

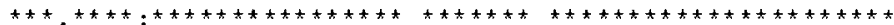

QECP YC RR TF SC YY SI KRHF ODKHE QS D TL YVCEFC HRRY RT KN SL TTHKSL QHRG QECP YC RR TF SC YYSL KRHF QDKHE QS D TL YVCEFC HRRY RT KNSL TTHKSL QHRG QECP YC RR TF SC YYSL KRHFQDKHE QS D TL YVCEEC HR RY RTKNSL TTHKSL OHRG QECP YC RR TF SC YYSL KRHF QDKHE QS DTL YVCEFC HR RYRT KNSL TTHKSL OHRG

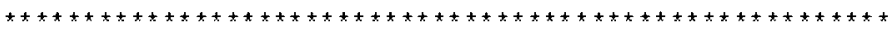

H RCD YC GKLL STKC TL KRH KE QQHW QR L N WA VC WLC HKYE RTLWSL WNHKS I YHRR HRCD VC GKLL ST WV TL KRH KE QOHL QPL N NA VC NLC HKVE RTL N SL WNHKS I YHRR HRCE VC GKLL STRL TL KRH TE QOHU QP L HSA RC SLC HKVF RTL N SL WNHKS I YTRR

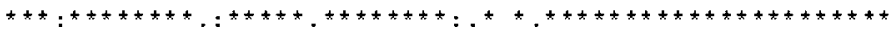
Core region

\begin{tabular}{|l|l|l|}
\hline BTB & BRcore & C2H2 \\
\hline
\end{tabular}

A

\begin{tabular}{|l|l|l|}
\hline $97 \%$ & $459 \mathrm{aa}$ & $96 \%$ \\
\hline
\end{tabular}

DmZ2

\begin{tabular}{|l|l|l|}
\hline BTB & BRcore & $\mathrm{C} 2 \mathrm{H} 2$ \\
\hline
\end{tabular}

$\mathrm{AaZ2}$

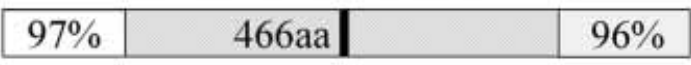

DmZ3

\begin{tabular}{|l|l|l|l|}
\hline BTB & BRcore & & $\mathrm{C} 2 \mathrm{H} 2$ \\
\hline
\end{tabular}

$\mathrm{AaZ3}$

\begin{tabular}{|l|l|l|}
\hline $97 \%$ & $466 \mathrm{a}$ & $100 \%$ \\
\hline
\end{tabular}

DmZ4

\begin{tabular}{|l|l|l|l|}
\hline BTB & BRcore & C2 $2 \mid$ \\
\hline
\end{tabular}

$\mathrm{AaZ4}$

\begin{tabular}{|l|l|l|l|}
\hline $97 \%$ & $459 \mathrm{aa}$ & $98 \%$ & \\
\hline
\end{tabular}

Figure 1 Alignment of predicted amino acid sequences of Aedes aegypti (Aa), Anopheles gambiae (Ag), Drosophila melanogaster (Dm) and Manduca sexta (Ms) BR. (A) Alignment of the BTB domains. (B) Alignment of the zinc-finger domains. The conserved Cys and His residues in the zinc-finger domains are highlighted in bold. (C) Schematic representation of sequence identity of structural domains between AaBR and DmBR. The predicted amino acid (aa) sequences of Aedes aegypti BR were compared with the GenBank database using a BLAST search. GenBank accession numbers for the cDNA sequences of Z1, Z2, Z3 and Z4 are AY499537, AY499538, AY499539 and AY499540 respectively. *, identical residues; :, conserved substitutions; ., semi-conserved substitutions. 
construct $\mathrm{pVg} 2 \cdot 1-\mathrm{luc}$. The construction of plasmids pAc5-AaEcR and pAc5-AaUSP has been described elsewhere (Wang et al. 1998).

The Drosophila L57-3-11 cell line (a kind gift from Dr Lucy Cherbas, Indiana University, USA) was maintained at $26-28{ }^{\circ} \mathrm{C}$ in Schneider Drosophila medium and supplemented with $5 \%$ fetal bovine serum. L57-3-11 is an EcR-deficient clone, derived from the Kc167 cell line by parahomologous targeting ( $\mathrm{Hu}$ et al. 2003). Transfection was conducted using CellFECTIN (Invitrogen) with an optimal DNA-lipid ratio of 1:4 (w/v), following the manufacturer's instructions. Typically, increasing amounts of BR (100, 300 and $900 \mathrm{ng}), 100 \mathrm{ng}$ pVg2·1-luc, $100 \mathrm{ng}$ pRLCMV/renilla luciferase (Promega) and CellFECTIN were mixed in a 24-well plate with a total volume of $250 \mu \mathrm{l}$ serum-free medium, and incubated at room temperature for $20 \mathrm{~min}$. The expression vector pAc5 $1 /$ V5/HisA was used as carrier DNA so that each well received an equal amount of total DNA. Renilla luciferase served as an internal control for transfection efficiency. The cells transfected with empty expression vectors $\mathrm{pAc} 5 \cdot 1 / \mathrm{V} 5 /$ HisA were used as a negative control. The transfection cocktail was added to L57-3-11 cells for $6 \mathrm{~h}$ at $27^{\circ} \mathrm{C}$. Transfection mixtures were then removed, replaced with fresh growth media, and allowed to incubate with or without ecdysteroid application $\left(2 \times 10^{-6} \mathrm{M}\right)$. After $36-48 \mathrm{~h}$ of incubation, the medium was aspirated and cells were lysed in $100 \mu \mathrm{l}$ passive lysis buffer (Promega). Dual luciferase activities were measured by Lumimark (Bio-Rad). The relative luciferase activity was obtained by normalization of the firefly luciferase activity against renilla luciferase activity.

Western blot analyses were carried out with a fraction of the L57-3-11 cells transiently transfected as described above. Twenty microliters of each sample were separated by SDS-PAGE and tested independently with antibodies against AaEcR, AaUSP and V5 (Invitrogen).

\section{Results}

\section{Cloning Aedes aegypti BR isoforms}

The four isoforms Z1, Z2, Z3 and Z4 were cloned from Aedes aegypti, utilizing a PCR-based strategy. These isoforms share an N-terminal core region of 459 amino acids, except that isoforms Z2 and Z3 contain seven more amino acids at position 272

\section{Z1 $\quad$ Z2 $233 \quad$ Z4}

$250 \mathrm{kDa}^{-}$

$150 \mathrm{kDa}-$

$100 \mathrm{kDa}-$

$75 \mathrm{kDa}-$

\section{$50 \mathrm{kDa}-$}

\section{$37 \mathrm{kDa}-$}

Figure 2 In vitro translation analysis. The cDNA sequences encoding AaBR Z1, Z2, Z3 and Z4 were translated by a coupled in vitro transcription-translation system and labeled by [ ${ }^{35} \mathrm{~S}$ ]methionine. Translation products were separated by SDS-PAGE using a 10\% gel followed by exposure to X-ray film. Molecular weight standards are shown on the left-hand side in decreasing order.

(Fig. 1G). The regions between the N-terminal BTB domain and the C-terminal zinc-finger domain were divergent among the four isoforms and showed no similarity to $D$. melanogaster, $A$. gambiae and M. sexta BR. Among the isoforms, the $\mathrm{Z1}$ and $\mathrm{Z4}$ zinc-finger domains showed a high degree of homology. This suggests that they diverged most recently and may have similar functions. The Z1, Z2, Z3 and Z4 proteins have a predicted molecular mass of $67 \mathrm{kDa}$ (561 amino acids (aa)), $64 \mathrm{kDa}(530 \mathrm{aa}), 73 \mathrm{kDa}(607$ aa) and $73 \mathrm{kDa}(607$ aa) respectively. The conceptual translation of AaBR isoforms was generally supported by in vitro-coupled transcription and translation assay (Fig. 2).

\section{Expression of $b r$ in the fat body and ovary of Aedes aegypti females}

In order to examine temporal and spatial expression patterns of the BR isoforms, RT-PGR 


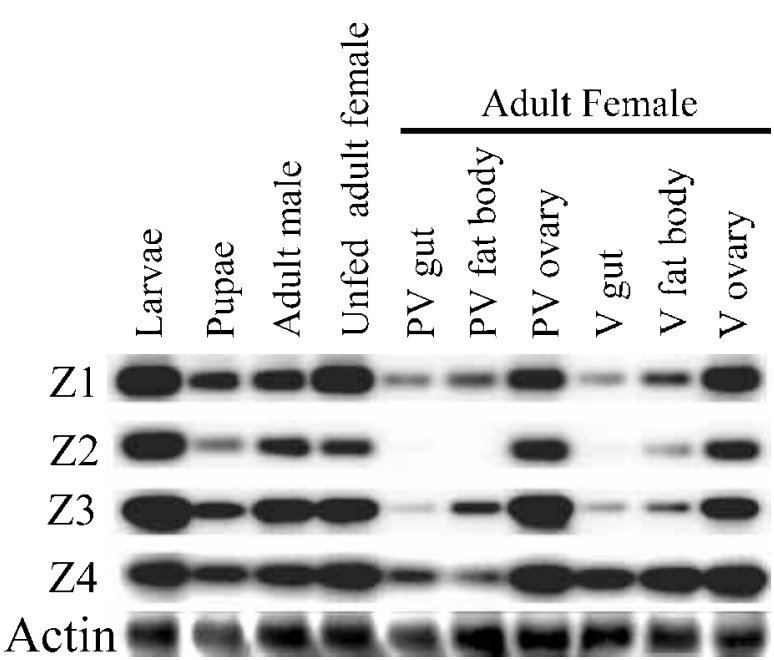

Figure 3 Tissue- and stage-specific expression of $A$. aegypti $\mathrm{BR}$ isoforms. Total RNAs were isolated from early 4th instar larvae (12-24 h post 3rd molt), early pupae (12-24 h post pupation), adult male (4 days post eclosion), unfed (PV) female mosquitoes (4 days post eclosion) and blood-fed (V) adult female mosquitoes (24 h PBM), and RT-PCR was performed. Isoform-specific probes were used to detect $A a B R$ expression by Southern blot. Actin RNA level is shown as a control.

experiments were first performed with total RNAs isolated from mosquitoes at different developmental stages. Relatively abundant transcripts of Z1, Z3 and $\mathrm{Z} 4$ isoforms were found in early 4th instar larvae $(12-24 \mathrm{~h}$ post 3rd molt), early pupae (12-24 h post pupation), adult male and unfed female mosquitoes, while Z2 mRNA was present to a lesser extent. In blood-fed adult female mosquitoes, expression of Z1, Z2 and Z4 in the fat body was enhanced after blood feeding ( $24 \mathrm{~h}$ PBM), while in ovary or gut, mRNA levels of BR isoforms at $24 \mathrm{~h}$ PBM were visibly changed as compared with those without blood feeding (Fig. 3).

To determine the expression profiles of BR isoforms in detail during vitellogenesis, real-time PCR was applied to measure the mRNA levels of the $\mathrm{BR}$ isoforms. In the fat body, $\mathrm{Z} 1$ transcripts showed a peak at the previtellogenic 84-96 h period (Fig. 4A). After a blood meal, Z1 transcripts gradually increased again and reached a second peak at $24 \mathrm{~h}$ PBM. Z4 mRNA displayed a similar profile to the $\mathrm{Zl}$ transcript; it was, however, considerably more abundant than Z1 mRNA and had a larger peak at $24 \mathrm{~h}$ PBM. The Z2 transcript level also increased during the previtellogenic period; however, it exhibited a sharp rise after the onset of vitellogenesis and reached an early peak at $8 \mathrm{~h} \mathrm{PBM}$ and the level of Z2 mRNA declined after that and rose again at $24 \mathrm{~h}$. Z3 transcript in the fat body remained at a relatively low level throughout the vitellogenic period, having two small peaks of expression during the previtellogenic period (84$96 \mathrm{~h}$ ) and at $24 \mathrm{~h} \mathrm{PBM}$. The expression of BR isoforms in the fat body returned to basal levels by 36-40 h PBM (Fig. 4A).

In the ovary, expression of $\mathrm{Z} 1$ and $\mathrm{Z} 4$ isoforms was augmented after blood feeding (Fig. 4B). mRNAs of both isoforms were abundant between 6 and $24 \mathrm{~h}$ PBM, reaching a broad peak between 16 and $24 \mathrm{~h} \mathrm{PBM,} \mathrm{then} \mathrm{gradually} \mathrm{declining} \mathrm{and}$ completely disappearing by $36 \mathrm{~h}$ PBM. Z2 expression reached a peak by $6 \mathrm{~h} \mathrm{PBM}$ and then declined to the background level by $36 \mathrm{~h}$ PBM. Interestingly, Z3 transcript was most abundant in ovaries of newly emerged mosquitoes, vanishing by $24 \mathrm{~h}$ post eclosion (Fig. 4B).

Transcripts of BR isoforms were examined by Northern blot analysis. Total RNA derived from mosquitoes at $24 \mathrm{~h}$ PBM was subjected to Northern blot analysis by using probes corresponding to the isoform-specific region of BR. Results showed that $\mathrm{Z} 1$ and $\mathrm{Z} 4$ isoforms were each represented by only one transcript, while $\mathrm{Z} 2$ and $\mathrm{Z} 3$ each had two transcripts, of unequal size. All transcripts were of different size, and each was larger than $9.5 \mathrm{~kb}$ (Fig. 5).

\section{Hormonal responses of mosquito br gene in mosquito fat body culture}

In the mosquito fat body, early peaks of BR transcript levels occurred during the previtellogenic period, which is under the control of JH III ( $\mathrm{Zhu}$ et al. 2003b). We tested whether the br gene is directly responsive to this hormone, using fat body culture. We cultured fat bodies of female mosquitoes 2 days after eclosion, which corresponded to the beginning of the previtellogenic rise in BR transcript levels, in the presence of JH III $\left(1 \times 10^{-6} \mathrm{M}\right)$ or with solvent (acetone) alone. We examined the responsiveness of $b r$ to JH III in fat bodies cultured for 4 and $8 \mathrm{~h}$. Total RNA was isolated from these samples and transcripts of BR isoforms were measured using real-time PCR. Figure 6 shows that after $8 \mathrm{~h}$ of incubation in the presence of JH III, transcript levels of Z1, Z2 


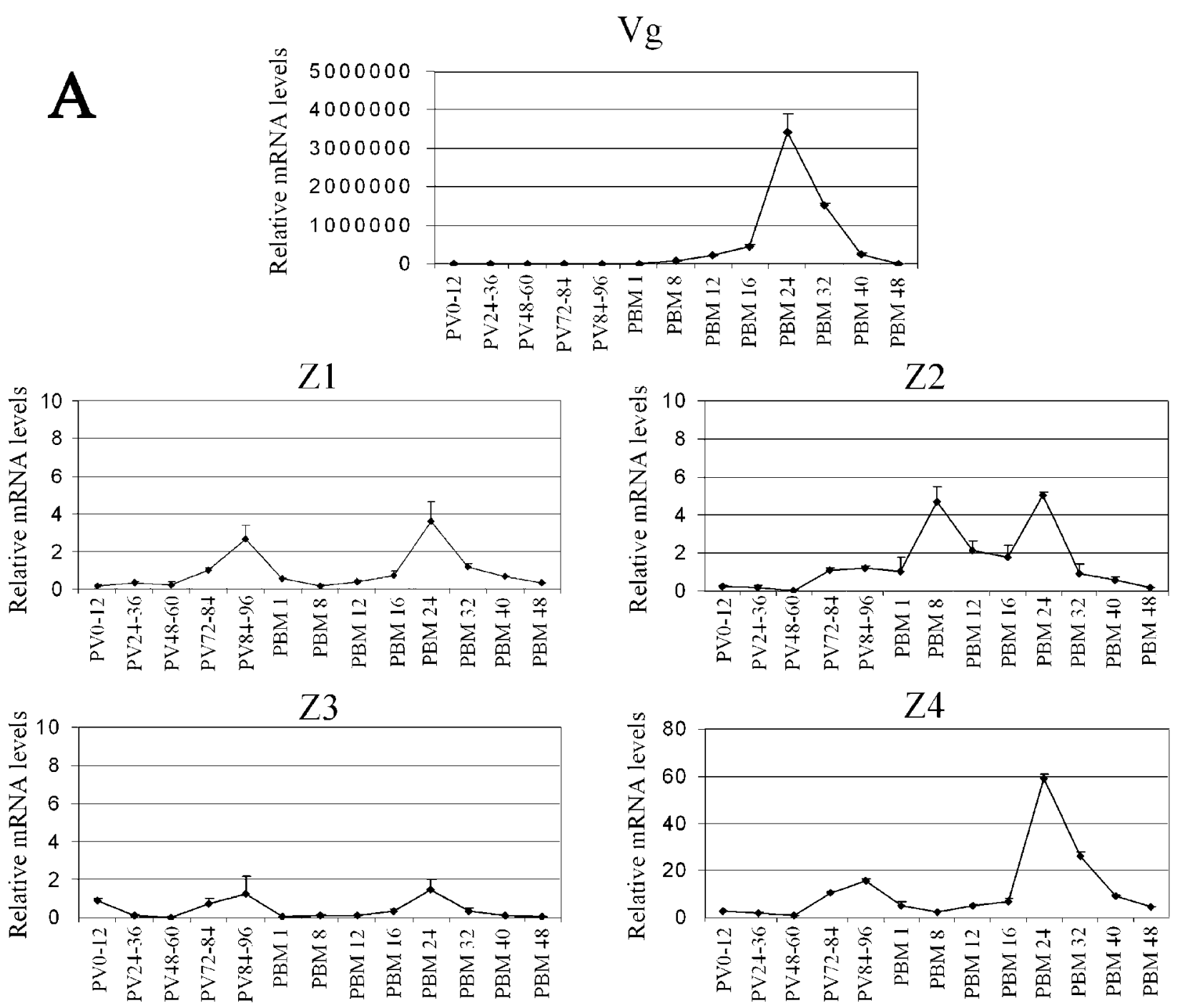

Figure $4(A)$.

and Z4 were considerably elevated. Z3 was not responsive to JH III in this experiment (not shown). Thus, the $b r$ gene is responsive to JH III during the $\mathrm{JH}$-dependent previtellogenic stage of mosquito fat body maturation.

The increase in BR isoform transcripts in both the fat body and ovaries after a blood meal suggested that $b r$ was induced by elevated ecdysteroid titers. We therefore examined the effect of $20 \mathrm{E}$ on the expression of BR isoforms utilizing an in vitro fat body culture system. Fat bodies were dissected from 3- to 4-day-old previtellogenic female mosquitoes, which are fully competent for $20 \mathrm{E}$ response, and cultured in medium with different concentrations of $20 \mathrm{E}$ for $4 \mathrm{~h}$. Total RNA was isolated from these samples and transcripts of BR isoforms were measured by real-time PCR. The analyses revealed that the mRNA levels of all four isoforms were stimulated by $20 \mathrm{E}$ in a dose-dependent manner (Fig. 7). The level of BR Z4 was dramatically increased, while lower levels were observed for transcripts for Z1, Z2 and Z3 isoforms. The BR transcripts reached their maximal expression with $10^{-7} \mathrm{M} 20 \mathrm{E}$.

Next, we examined the effect of $20 \mathrm{E}$ $\left(1 \times 10^{-6} \mathrm{M}\right)$ on the expression of $\mathrm{BR}$ isoforms during $16 \mathrm{~h}$ of culturing. $V g$ gene expression was monitored as a control for these in vitro fat body culture experiments. The $\mathrm{Vg}$ mRNA level increased in the presence of $20 \mathrm{E}$, but declined if $20 \mathrm{E}$ 
B
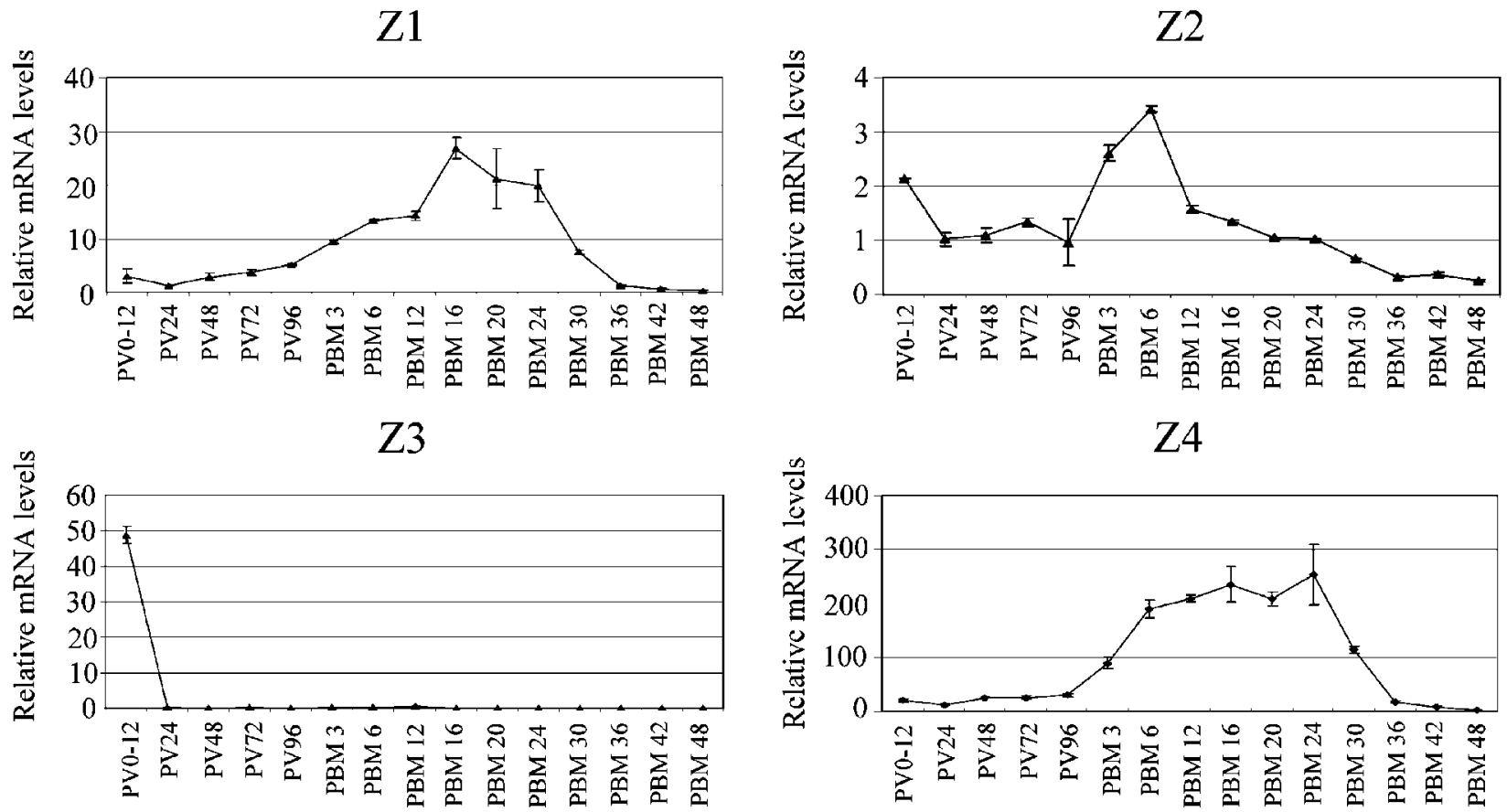

Figure 4 (B).

Figure 4 Developmental profiles of $A a B R$ in the fat body and ovary during vitellogenesis. Real-time PCR was performed to quantify mRNA levels of four BR isoforms in the fat body and ovary during vitellogenesis. Total RNA $(0.05 \mu \mathrm{g})$ of each sample was used for real-time PCR. The results are means of independent triplicate samples \pm S.D., normalized against actin expression. (A) Developmental profiles of $A a B R$ and $V g$ mRNA in the fat body during vitellogenesis. (B) Developmental profiles of $A a B R$ mRNA in the ovary during vitellogenesis.

was withdrawn at $4 \mathrm{~h}$ of incubation (Fig. 8A). Thus, the $V g$ gene required the continuous presence of $20 \mathrm{E}$ for its expression. In contrast, in fat bodies cultured in the constant presence of 20E levels, all four $\mathrm{BR}$ isoform transcripts increased during the first $4 \mathrm{~h}$ but then declined to a basal level (Fig. 8A). However, if $20 \mathrm{E}$ was withdrawn from the medium after the first $4 \mathrm{~h}$ of incubation, transcripts of all BR isoforms increased again. We found that mRNA levels of all four isoforms were greatly augmented by $\mathrm{CHX}$ alone at $4 \mathrm{~h}$ after the start of incubation (Fig. 8B). A similar phenomenon has been described in the Drosophila imaginal discs in vitro culture experiment, in which the $b r$ gene is repressed in the absence of ecdysteroid, and that repression requires protein synthesis (Bayer et al. 1996). When mosquito fat bodies were treated with both 20E and CHX, Z1 and Z4 mRNA increased over time to levels higher than in those treated with either 20E or CHX alone. The level of Z2 transcript was elevated after $8 \mathrm{~h}$ of incubation with
20E and CHX, but decreased with longer incubations. On the other hand, Z3 mRNA levels decreased to a basal level after $8 \mathrm{~h}$ of incubation with either CHX alone or both 20E and CHX. These results indicated that these isoforms were under distinct regulatory mechanisms. However, despite these differences the expression of $b r$ isoform transcripts in the mosquito fat body is under the control of a 20E negative feedback control, characteristic of 20E-regulated early response genes.

We also tested whether or not responsiveness to JH III was altered in the fat bodies which have already achieved competence for $20 \mathrm{E}$ responsiveness. We cultured fat bodies dissected from 3- to 4-day-old previtellogenic female mosquitoes. When JH III alone $\left(1 \times 10^{-6} \mathrm{M}\right)$ was added to the culture medium, transcription of $\mathrm{BR}$ isoforms was not elevated above the background levels after $16 \mathrm{~h}$ of incubation (Fig. 8C). However, if JH III and 20E were added together, $\mathrm{JH}$ prevented the rise in 


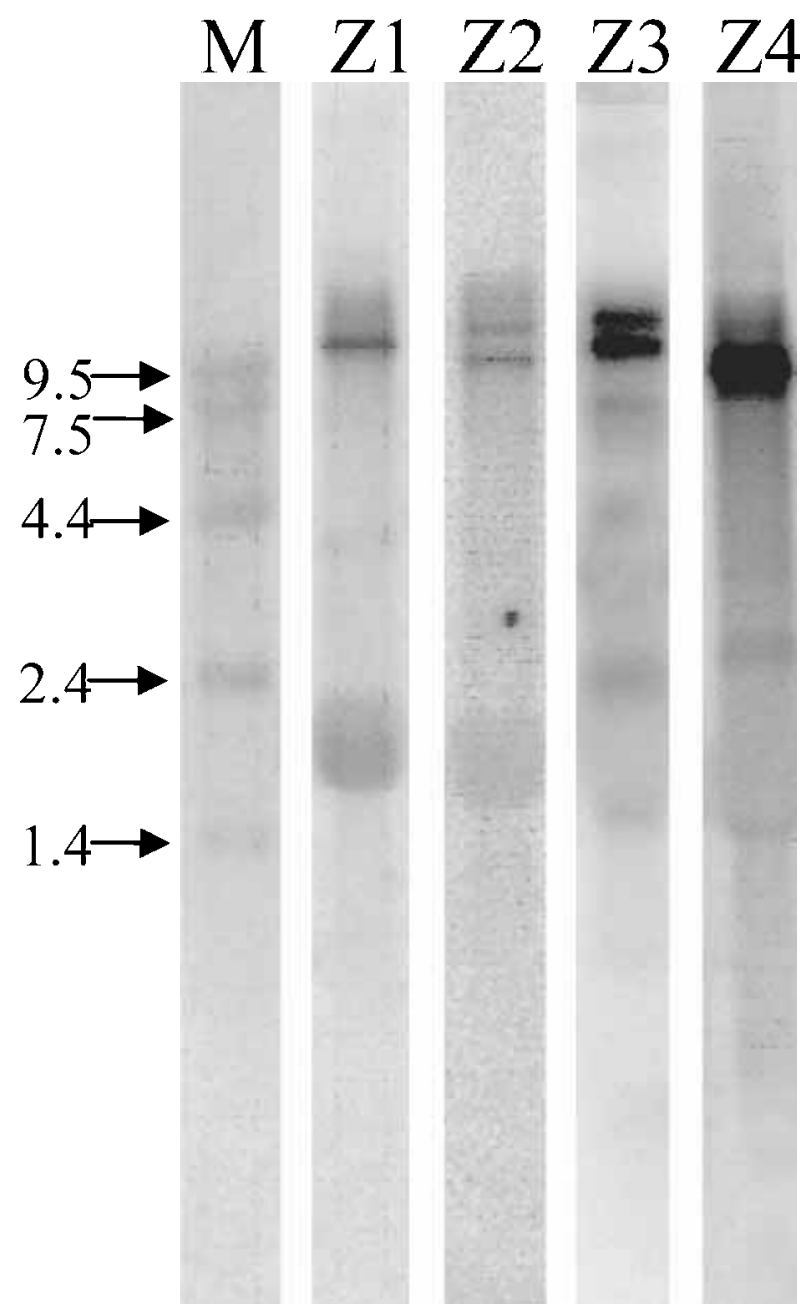

Figure 5 Northern blot analysis of $A a B R$ transcripts. Total RNA was extracted from adult mosquitoes. RNA $(10 \mu \mathrm{g})$ was loaded in each lane. Northern blots were hybridized with $A a B R$ isoform-specific probes. Lane M, RNA marker; isoform-specific probes used are shown at the top of each lane.

20E-induced expression of each of the BR isoforms that occurred at $4 \mathrm{~h}$ after the start of incubation. At later time-points of incubation, there was no apparent inhibitory effect of $\mathrm{JH}$ on $b r$ expression. This was in contrast to the effect of JH and 20E on $\mathrm{Vg}$ expression, where $\mathrm{JH}$ prevents the increase for at least $16 \mathrm{~h}$ (Fig. 8C, top panel).

\section{DNA-binding sites of BR isoforms in the vitellogenin gene $5^{\prime}$-regulatory region}

The potential binding sites of $\mathrm{BR}$ isoforms in the mosquito vitellogenin gene $(V g) \quad 5^{\prime}$-regulatory $\square \mathrm{CM} \square \mathrm{JH}$

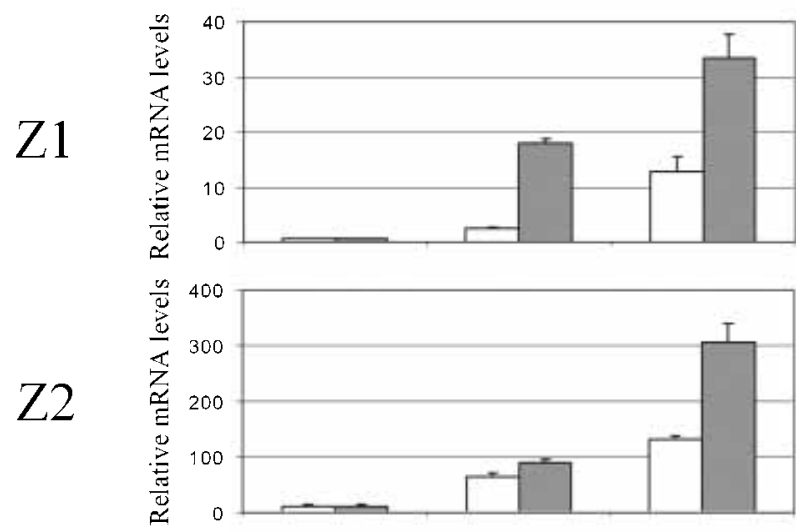

Z4

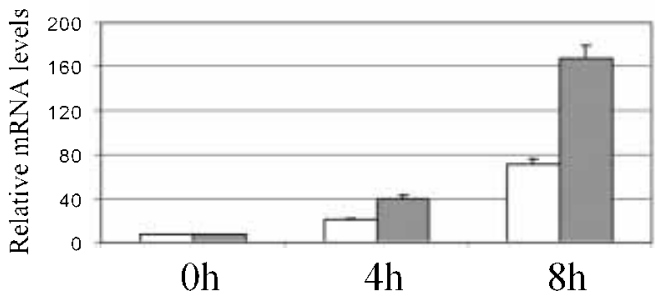

Figure $6 \mathrm{JH}$ III activates br gene expression. Fat bodies from 2-day-old female mosquitoes were exposed to $1 \times 10^{-6} \mathrm{M} \mathrm{JH}$ III (shaded bars) or acetone (open bars) in vitro for 4 or $8 \mathrm{~h}$. Total RNA was then isolated from these samples. Real-time PCR was performed using isoform-specific primers for AaBR Z1, Z2, Z3 (not shown) and $Z 4$. The results represent the means of independent triplicate samples \pm S.D., normalized against actin expression.

region, simply called here the $V g$ promoter, were screened with consensus DNA-binding sequences identified in Drosophila (von Kalm et al. 1994), and examined using EMSA. Double-stranded oligonucleotides containing the consensus BR isoformbinding sites were labeled with ${ }^{32} \mathrm{P}$ and used as probes. They formed specific DNA-protein complexes with in vitro translated BR isoforms, but not with the control reticulocyte lysate. Oligonucleotides containing distinct putative BR-binding sites in the $V g$ promoter were added to reactions as competitors. For example, two putative binding sites for isoform $\mathrm{Z1}, 1 \mathrm{~A}$ and $1 \mathrm{~B}$ were found in the $V g$ promoter. Interaction between a consensus sequence and $\mathrm{Zl}$ was competitively abolished by the 100-fold excess of $1 \mathrm{~A}$ site, but not by $1 \mathrm{~B}$ site (Fig. 9A). In addition, $1 \mathrm{~A}$ and $1 \mathrm{~B}$ sites were tested by direct binding of $\mathrm{Z} 1$, and the results were consistent with those of the competition binding experiments (data not shown). Similar competitive 

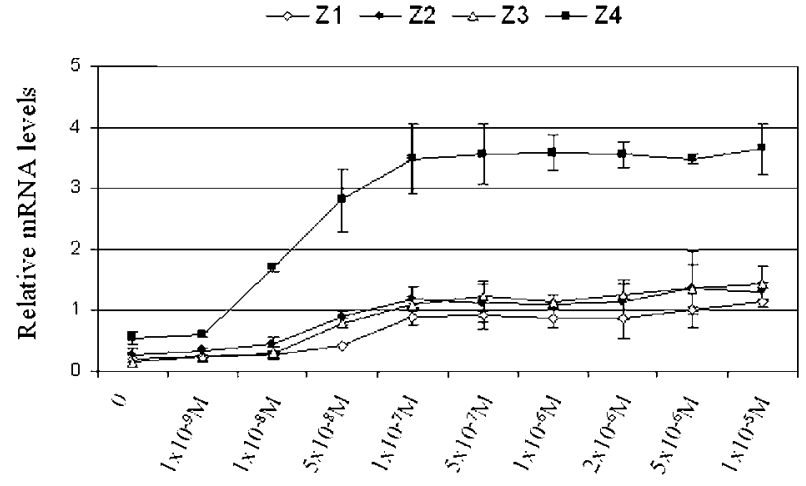

Figure $7 \mathrm{AaBR}$ response to $20 \mathrm{E}$ in fat body culture. The transcription of $A a B R$ in response to $20 \mathrm{E}$ was examined in an in vitro culture system. Fat bodies from previtellogenic female mosquitoes at 4 days post eclosion were incubated for $4 \mathrm{~h}$ at the $20 \mathrm{E}$ concentrations indicated. Total RNA was then isolated from these samples. Real-time PCR was performed using isoform-specific primers for $A a B R$ Z1, Z2, Z3 and $Z 4$. The results represent the means of independent triplicate samples \pm S.D., normalized against actin expression.

and direct binding experiments were performed with Z2, Z3 and Z4. We found two binding sites for $\mathrm{Z} 2$, three binding sites for $\mathrm{Z} 3$ and seven binding sites for Z4 in the $V g$ promoter (Fig. 9B, C and D). In vitro-expressed $\mathrm{Z} 4$ protein showed a high affinity to $4 \mathrm{~A}$ and $4 \mathrm{~F}$, but a low affinity to other potential binding sites (Fig. 9D). Direct binding experiments confirmed the results of competitive binding assays for Z2, Z3 and Z4 (data not shown). Potential binding sites for $\mathrm{BR}$ isoforms on the $5^{\prime}$-regulatory region $V g$ gene are shown in Fig. 10. The presence of these numerous potential binding sites suggests that BR isoforms are directly involved in 20Emediated regulation of the $V g$ gene during mosquito egg maturation.

\section{Different functions of BR isoforms on $\mathrm{Vg}$ promoter in Drosophila L57-3-11 cells}

To investigate the possible effect of BR isoforms on the $V g$ promoter, we used a cell transfection assay. The reporter plasmid $\mathrm{pVg} 2 \cdot 1$-luc was transfected into L57-3-11 cells, alone, with the expression plasmids of one of the BR isoforms or with AaEcR and AaUSP. When the reporter and individual BR isoform expression vectors were transfected into cells, no significant change of luciferase activity was detected (Fig. 11A). Co-transfection of $\mathrm{pVg} 2 \cdot 1-\mathrm{luc}$ and AaEcR/AaUSP resulted in a sevenfold increase of luciferase activity, when treated with 20E, compared with no 20E treatment (Fig. 11B lanes 3 and 4). When $\mathrm{Zl}$ was co-transfected along with AaEcR/AaUSP, no significant change of luciferase was detected without 20E treatment (Fig. $11 \mathrm{~B}$, lane 5). However, in the presence of $20 \mathrm{E}, \mathrm{Z1}$ repressed AaEcR/AaUSP-mediated activation of the $V g$ promoter. Increasing the amount of $\mathrm{BR}$ resulted in a further decrease in EcR/USPdependent transactivation of the reporter gene (Fig. 11B, lanes 6-8). Similar results were observed when $\mathrm{Z} 4$ was co-transfected with $\mathrm{pVg} 2 \cdot 1-\mathrm{luc}$ and AaEcR/AaUSP (Fig. 11B, lanes 17-20). In contrast, when $\mathrm{Z} 2$ was co-transfected with $\mathrm{pVg} 2 \cdot 1-$ luc and AaEcR/AaUSP, Z2 enhanced EcR/USPmediated activation of the reporter gene in the presence of $20 \mathrm{E}$ and showed no effect on the reporter gene in the absence of $20 \mathrm{E}$ (Fig. 11B, lanes 9-12). Z3 only marginally increased the effect of EcR/USP in the presence of 20E (Fig. 11B, lanes 13-16).

In order to demonstrate that the observed BR effects on the reporter gene were not due to alteration of AaEcR/AaUSP translation, Western blot analysis was performed. An aliquot of each sample $(20 \mu \mathrm{l}$ out of $100 \mu \mathrm{l})$ was examined with antibodies against AaEcR, AaUSP and V5 tag (Fig. 11C). All exogenous proteins were detected after transfection, and the endogenous Drosophila USP was seen as well. Importantly, levels of AaEcR and AaUSP proteins did not change significantly after co-transfection with BR plasmids. Thus, Western blot analysis indicated that BR acts as a true modulator of the ecdysteroid receptor action.

\section{Discussion}

In this study, we have shown that the $b r$ gene is involved in the $20 \mathrm{E}$ regulatory hierarchy controlling vitellogenesis in the mosquito $A$. aegypti. Our conclusion is substantiated by several lines of evidence. First, expression of Z1, Z2 and Z4 isoforms was stimulated after blood feeding in both vitellogenic tissues, the fat body and the ovary, corresponding to the peaks of ecdysteroid titers (Hagedorn et al. 1975). In the fat body, the mRNA profiles of these three isoforms correlated well with those of YPP genes, $V g, V C P$ and $V C B$ (Cho et al. 1991, 1999, Cho \& Raikhel 1992). Secondly, these 
A
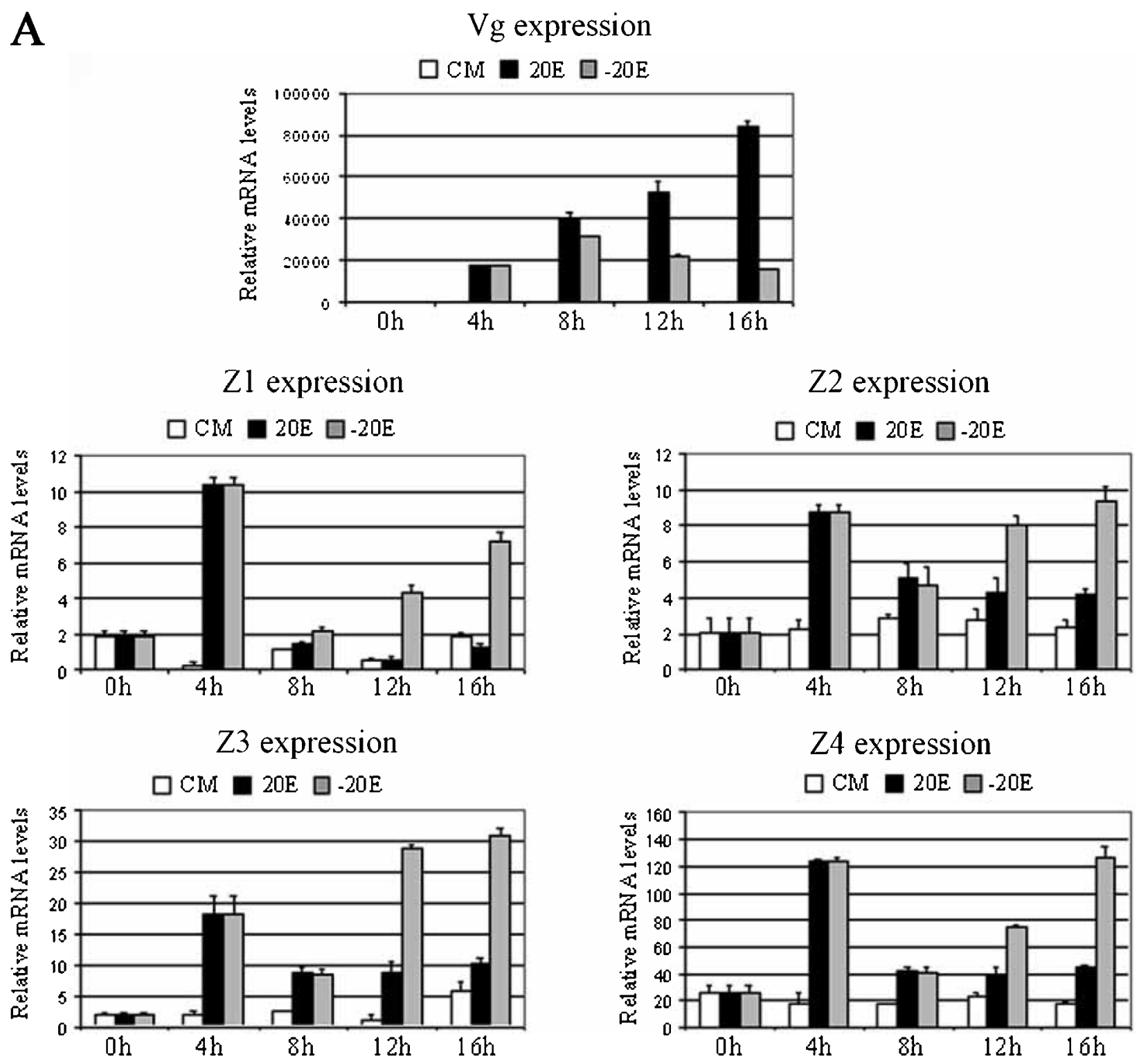

Figure $8(A)$.

BR isoforms were activated by $20 \mathrm{E}$ in an in vitro fat body system and behaved as early genes, having a negative autoregulatory loop, which could be blocked by the protein inhibitor CHX. Thirdly, multiple binding sites for all four BR isoforms were present in the $5^{\prime}$-regulatory region of the $V g$ gene. Finally, effects of BR isoforms on $V g$ gene expression have been demonstrated by cell transfection analysis.

The BR sequence analyses have shown that the BTB domain and isoform-specific DNA-binding domains are highly conserved in BR from A. aegypti,
A. gambiae, D. melanogaster, and $M$. sexta. In Drosophila, there are six different isoforms, four of which vary in the zinc-finger domains. Three isoforms have been found in Manduca (Z2, Z3 and $\mathrm{Z4)}$ and in Anopheles gambiae (Z1, Z2 and Z3). The $\mathrm{BR}$ isoforms have various $\mathrm{C} 2 \mathrm{H} 2$ zinc-finger regions that can bind to different $\mathrm{A} / \mathrm{T}$-rich consensus sequences. The high level of variability of these A/T-rich sequences suggests that additional determinants are important for specific DNA recognition. The $\mathrm{BTB} / \mathrm{POZ}$ domain that is present in all $\mathrm{BR}$ isoforms appears to be such a 
B
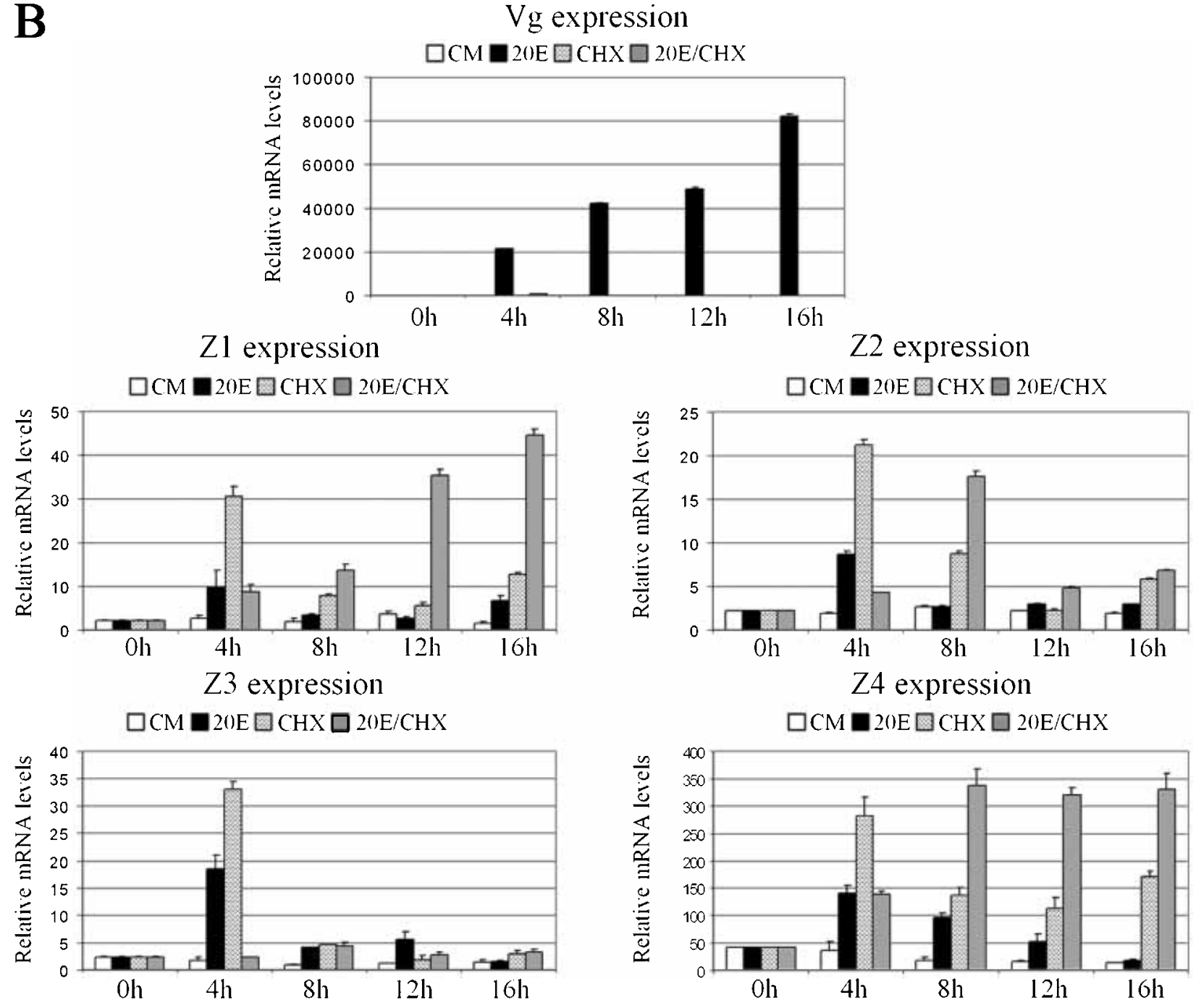

$\square \mathrm{CM}$ 20E $\mathrm{CHX} \square 20 \mathrm{E} / \mathrm{CHX}$

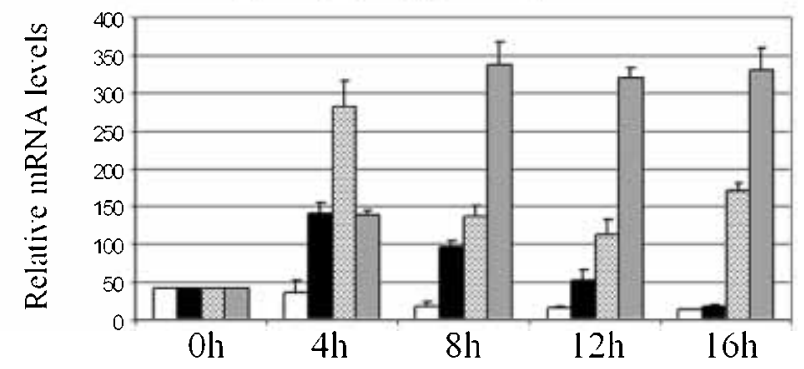

Figure 8 (B).

determinant. The crystal structure of the BTB domain of promyelocytic leukemia zinc finger was determined and revealed to form a homodimer (Ahmad et al. 1998). The domain mediates a functionally relevant dimerization in vivo, promoting strong co-operative DNA binding to multiple sites (Bardwell \& Treisman 1994). The BTB/POZ domains are thus likely to play a critical role in targeting proteins to specific chromosomal loci.

The presence of the zinc-finger motifs suggests that BR action is mediated by direct sequencespecific interactions with DNA. Based on the use of recombinant Drosophila BR isoforms produced in $E$. coli for in vitro footprint experiments, several studies have reported a direct in vitro binding of these proteins to promoter regions of the BR-regulated Sgs4 (Von Kalm et al. 1994), L71 (Crossgrove et al. 1996), Fbp1 (Mugat et al. 2000) and the first introns of the Dopa decarboxylase $(D d c)$ gene (Hodgetts et al. 1995). In the salivary gland of Drosophila, both the BR-Z1 and E74A proteins contribute to $L 71$ late gene activation and directly regulate L71-6 (Fletcher \& Thummel 1995a, Urness \& Thummel 1995). Because one of the E74A-binding sites at L71-6 overlaps with BR Z1-binding sites 1 and 2, it is possible that direct interactions between these proteins are necessary for L71-6 activation. Likewise, multiple putative binding sites of $A a \mathrm{BR}$ are found in the mosquito $\mathrm{Vg}$ gene, and these binding sites are spread over $2 \cdot 1 \mathrm{~kb}$ 
$\mathrm{C}$
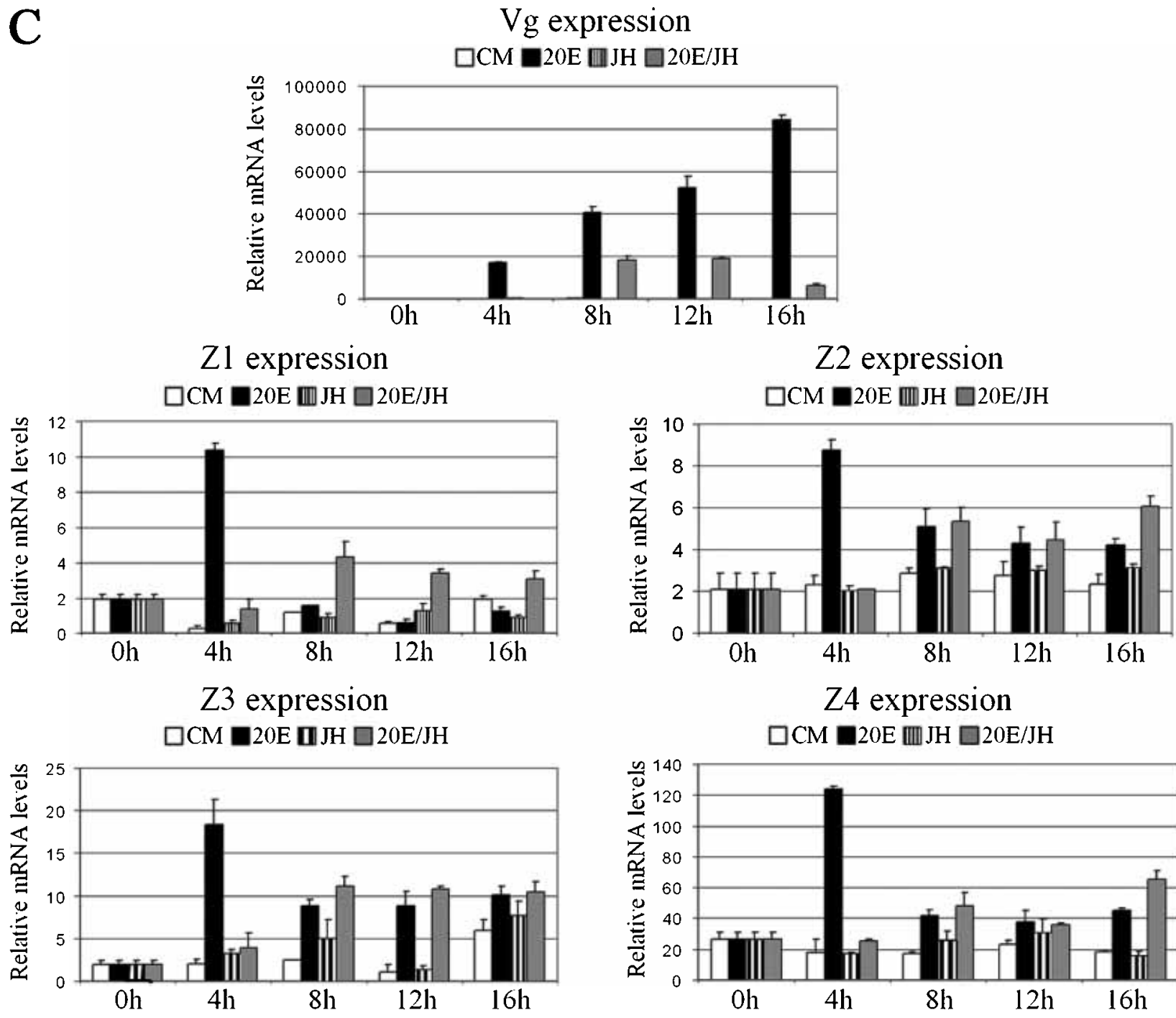

Figure 8 Effects of 20E, CHX and JH III on $A a B R$ expression in the fat body cultured in vitro. Previtellogenic fat bodies dissected from female mosquitoes (4 days after eclosion) were incubated for $16 \mathrm{~h}$ under the different treatments indicated in the diagram. $V g$ gene expression was included as a control. (A) Effects of 20E on $A a B R$ expression. CM, culture medium; 20E, medium with $2 \times 10^{-6} \mathrm{M} \mathrm{20E} ;-20 \mathrm{E}, 4 \mathrm{~h}$ in medium with $2 \times 10^{-6} \mathrm{M} 20 \mathrm{E}$ and followed by $12 \mathrm{~h}$ without $20 \mathrm{E}$. (B) Effects of $\mathrm{CHX}$ on $20 \mathrm{E}$-induced $\mathrm{AaBR}$ expression. $\mathrm{CHX}$, medium with $1 \times 10^{-5} \mathrm{M}$ $\mathrm{CHX}$; 20E/CHX, medium with $2 \times 10^{-6} \mathrm{M} 20 \mathrm{E}$ and $1 \times 10^{-5} \mathrm{M} \mathrm{CHX}$. (C) Effects of JH III on 20E-induced AaBR expression. $\mathrm{JH}$, medium with $1 \times 10^{-6} \mathrm{M} \mathrm{JH} \mathrm{III;} 20 \mathrm{E} / \mathrm{JH}$, medium with $2 \times 10^{-6} \mathrm{M} 20 \mathrm{E}$ and $1 \times 10^{-6} \mathrm{M} \mathrm{JH}$ III. RNA was extracted from fat bodies and subjected to real-time PCR. The results show the means of independent triplicate samples \pm S.D., normalized against actin expression. Each panel represents the expression of different genes, which are labeled at the top of each panel.

of its 5 -regulatory region, with some binding sites close to those of E75 and E74 (Fig. 10). Gel shift assays confirmed that AaBR isoforms bind to these sites in vitro, suggesting that $\mathrm{BR}$ isoforms may function on the $\mathrm{Vg}$ promoter by direct binding.
In both Manduca and Drosophila, ecdysteroid induces and co-ordinates molting, and $\mathrm{JH}$ controls some characteristics of the molt. In both species, it is the appearance of $b r$ transcripts in the final instar that correlates with commitment from larval to pupal differentiation. These transcripts are 
A

\section{Z1 TNT \\ Cold probe}

Non specific competitor

Specific competitor
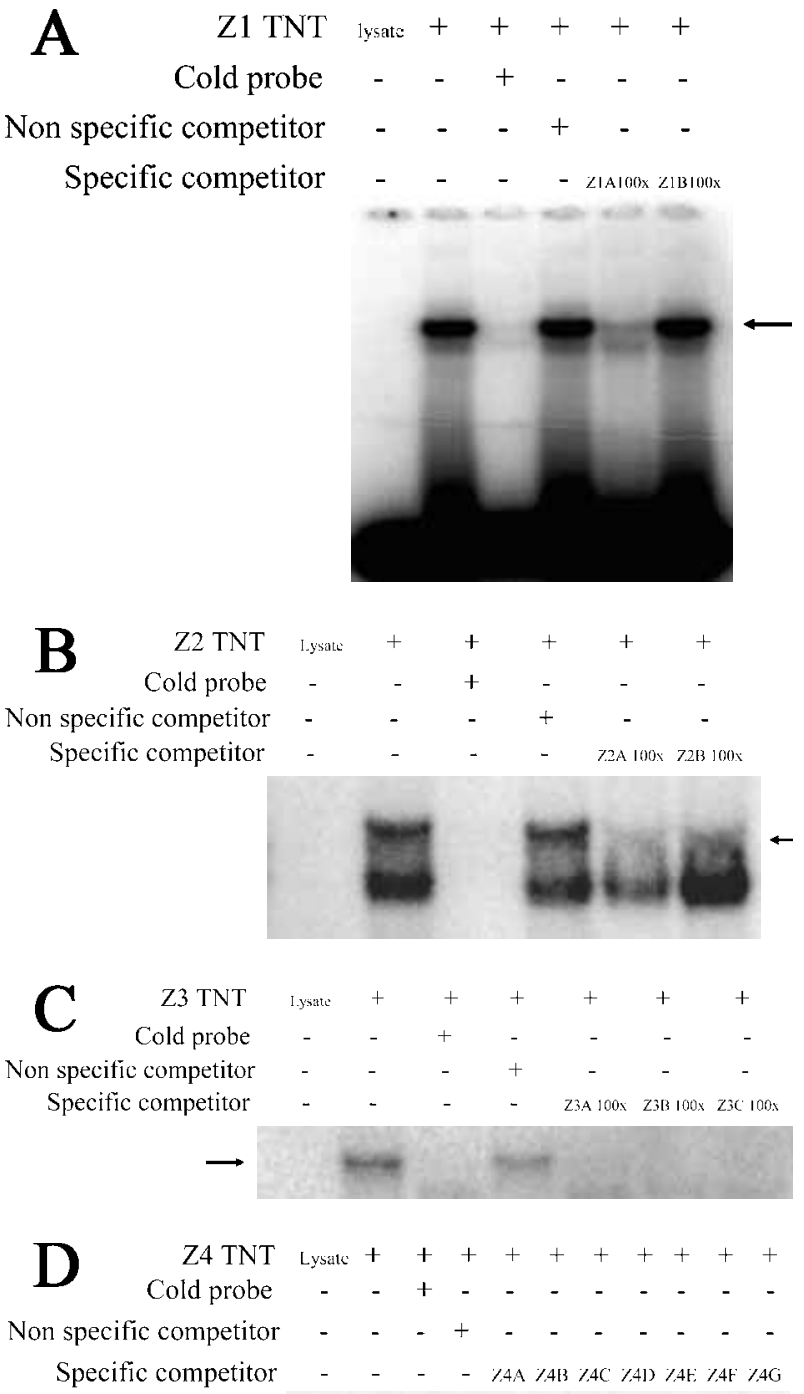

Figure 9 Competitive EMSA analyses of AaBR-binding properties to various binding sites in the $V g$ promoter. Drosophila BR consensus DNA-binding sequences were labeled as probes, and EMSA was performed with in vitro-translated (A) AaBR Z1, (B) Z2, (C) Z3 and (D) Z4. A 100 -fold molar excess of unlabeled potential AaBR DNA-binding sequences was added as specific competitors. Arrows indicate the specific retardation complexes. TNT, in vitro coupled transcription and translation. normally induced by $20 \mathrm{E}$, and $\mathrm{JH}$ blocks their appearance in larval Manduca (Zhou \& Riddiford 2002). In contrast, juvenoid treatment of a young pupa of Manduca or the white puparium of Drosophila results in the reappearance of $\mathrm{BR}$ leading to the formation of a Manduca second pupa or a Drosophila second pupal abdomen (Zhou \& Riddiford 2002). In our experiments with in vitro culture of fat bodies from previtellogenic female mosquitoes, we detected different effects of JH III on the expression of $b r$, depending on the age of females. The fat body reaches its complete $20 \mathrm{E}$ responsiveness by $72 \mathrm{~h}$ after eclosion, when $20 \mathrm{E}$ is capable of eliciting the highest level of activation of both early and late genes (Zhu et al. 2003b). Significantly, the $b r$ gene was responsive to JH III, which activated its expression in 2-day-old (48 h post eclosion) females. In contrast, $b r$ was not responsive to JH III alone in the fat bodies of fully competent mosquitoes (3-4 days post eclosion). In these fat bodies, however, 20E induction of $b r$ expression was blocked by JH III. Thus, $b r$ is responsive to $\mathrm{JH}$ activation at the previtellogenic stage when the reproductive tissues are preparing for 20E-regulated vitellogenesis. After reaching the stage of complete competence to $20 \mathrm{E}, b r$ responsiveness to $\mathrm{JH}$ is switched off. Further studies should elucidate the molecular mechanism underlying this switch in $b r$ responsiveness to $\mathrm{JH}$.

In the mosquito fat body, the early genes $E 74$ and E75 are expressed only after the initiation of vitellogenesis and an increase in ecdysteroid titers (Pierceall et al. 1999, Sun et al. 2002). In contrast, levels of all $\mathrm{BR}$ isoform transcripts increased by the completion of the previtellogenic stage, indicating that the presence of $\mathrm{BR}$ is important for activation of 20E-regulated target effector genes. Our preliminary gel-shift analysis of fat body nuclear extracts indicated the presence of active BR proteins in previtellogenic females (L Chen, J Zhu \& A S Raikhel, unpublished results). This situation is similar to that with EcR and USP proteins, which are also present in the previtellogenic fat body nuclei (Zhu et al. 2003b).

The PBM profile of AaBR Z2 mRNA in the fat body is reminiscent of those of E75 isoform transcripts, which exhibit early and late peaks (Pierceall et al. 1999). The early peak of expression is also characteristic for AaEcRB (Wang et al. 2002). The timing of these early peaks closely correlates with the first small peak of the ecdysteroid titer 


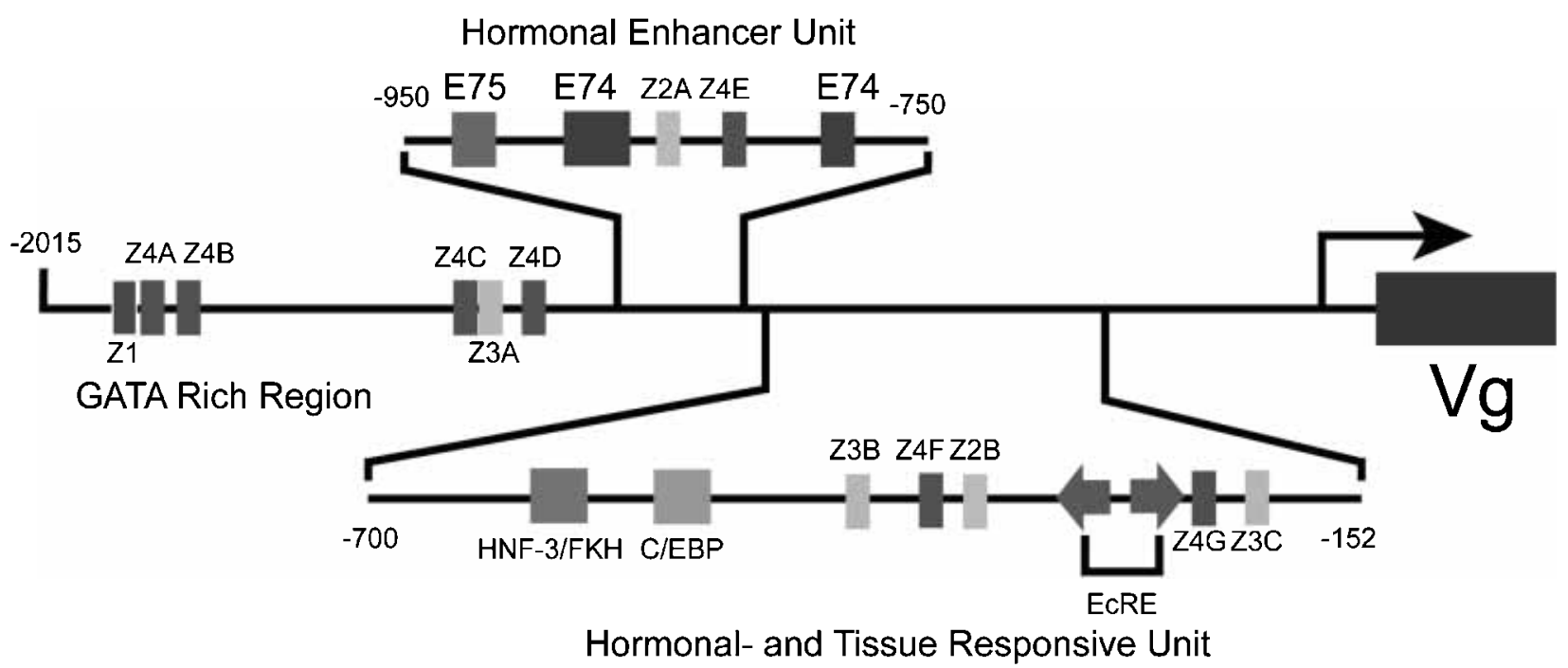

Figure 10 Schematic illustration of the AaBR-binding sites in the $\mathrm{Vg}$ promoter. Hormonal- and tissue-responsive unit (EcR-USP, HNF-3/FKH, C/EBP-binding sites), hormonal enhancer unit (E74, E75 -binding sites), and GATA-rich region are presented in the $V g$ promoter, to show the context of the BR isoform-binding sites. HNF-3/FKH, hepatic nuclear factor-3/forkhead; C/EBP, CCAAT enhancer-binding protein, ECRE, ecdysone response element.

in vitellogenic female mosquitoes, $4-6 \mathrm{~h}$ PBM (Hagedorn et al. 1975). In contrast, during the vitellogenic period, AaBR Z1, Z3 and Z4 transcripts have only one late peak at 24-32 h $\mathrm{PBM}$ that correlates with the expression profiles of $A a E 74 B, A a U S P B$ and the YPP genes, $V g, V C P$ and VCB (Cho \& Raikhel 1992, Cho et al. 1999, Wang et al. 2000, Sun et al. 2002).

Despite differences in expression profiles in vivo, mRNA transcripts of all four isoforms accumulate rapidly in the fat body in response to $20 \mathrm{E}$, but decline after $4 \mathrm{~h}$ when tested for induction in tissue culture. The sensitivity of BR transcripts to $20 \mathrm{E}$ are similar to those of other early genes $A a E 74 B$ and $A a E 75 B$ as well as to $A a U S P B$, which all reach their maximal expression at $10^{-7} \mathrm{M} 20 \mathrm{E}$ (Pierceall et al. 1999, Wang et al. 2000, Sun et al. 2004). In contrast, the $20 \mathrm{E}$ concentration required for maximal induction of the target genes, $V C P$ and $V g$, is 10-fold higher (Deitsch et al. 1995). Interestingly, AaE74 isoform mRNAs, which also have very different expression profiles in the fat body, respond simultaneously to $20 \mathrm{E}$ activation in the fat body culture in vitro (Sun et al. 2002, 2004). The differences between in vivo mRNA profiles of $\mathrm{BR}$ and E74 isoforms and their in vitro 20E induction in organ culture are probably due to the lack of a complete set of humoral factors in the culture medium, which are present in the hemolymph of vitellogenic females. Although the identity of such additional factors remains to be determined, involvement of the CREB transcription factor in the termination of $V g$ gene expression strongly supports the action of a peptide hormone on the fat body during late vitellogenesis (Dittmer et al. 2003). In vivo action of such additional hormones can affect the expression patterns of individual early genes in a unique way. It is also possible that the remaining $\mathrm{JH}$ present after blood feeding causes the $b r$ expression delay in vivo.

In Drosophila, the $b r$ gene is crucial at several stages of oogenesis (Deng \& Bownes 1997). Its expression regulates endoreplication of the polyploid follicle cells, specific amplification of the chorion genes and later positioning of the chorionic appendages. All BR isoforms are expressed in oogenesis, while $\mathrm{Z1}$ is the sole isoform expressed at high levels in the appendage-producing cells (Tzolovsky et al. 1999). The early induction of $b r$ is controlled by 20E, similar to its expression during metamorphosis as an early ecdysteroid-response gene. mRNA expression of AaBR isoforms Z1, Z2 and $\mathrm{Z} 4$ in the ovary is induced after a blood meal and has different profiles during vitellogenesis. This suggests that AaBR may display similar functions during oogenesis in $A$. aegypti.

Individual BR isoforms play distinct roles during molts and metamorphosis in D. melanogaster and $M$. 


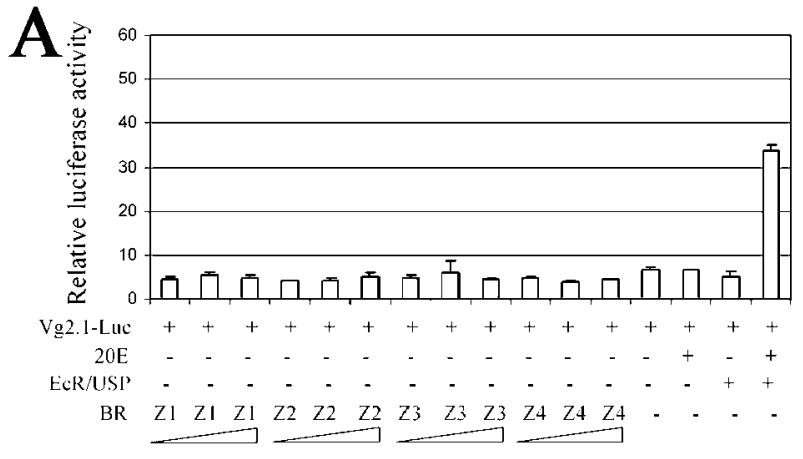

B
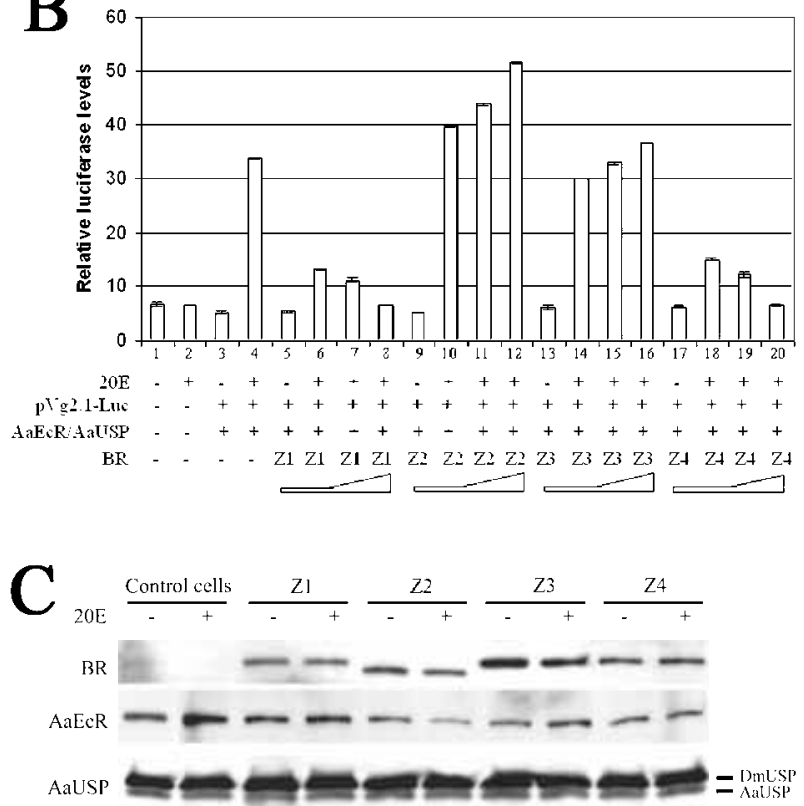

Figure $11 \mathrm{BR}$ isoforms display different functions on the $V g$ promoter in transfection assays. (A) Effect of BR alone on the $V g$ promoter. In the presence or absence of $20 \mathrm{E}$, the $\mathrm{pVg} 2 \cdot 1-\mathrm{luc}$ reporter plasmid (100 $\mathrm{ng}$ ) was co-transfected into L57-3-11 cells with increasing amounts of $Z 1, Z 2, Z 3$ and $Z 4$. A renilla luciferase expression vector pRLCMV (Promega; $100 \mathrm{ng}$ ) was co-transfected as an internal control, and firefly luciferase activity was normalized to renilla luciferase activity (relative luciferase activity). The results represent an average of three independent experiments \pm S.D. (B) BR modulates the transactivation of the $V g$ promoter by $A a E c R / A a U S P$. The experiment was performed as described above, except that L57-3-11 cells were co-transfected with expression vectors of $\mathrm{BR}$ isoforms, AaEcR (100 ng), and AaUSP $(100 \mathrm{ng})$. (C) Western blot analysis with the transfected L57-3-11 cells treated with or without 20E. Antibodies against V5 tag, AaEcR and AaUSP were used in the Western blot and are labeled on the left-hand side. Transfected BR isoforms are indicated at the top of the panel. sexta. In the imaginal discs and the abdominal epidermis of Drosophila during the formation of the pupal cuticle, $\mathrm{Zl}$ is the predominant isoform and is needed for pupal cuticle formation (Bayer et al. 1996, 1997). In contrast, Z4 is predominant in the epidermis during pupal cuticle formation in $M$. sexta (Zhou et al. 1998, Zhou \& Riddiford 2001). The Manduca Z4 transgene can partially substitute for Drosophila Z1 in the Drosophila $r b p^{1}$ mutant (Bayer et al. 2003). Likewise, Z4 expression is predominant in the fat body and ovary of the adult $A$. aegypti females. During the molt to the final larval instar in Manduca, misexpression of $\mathrm{Z} 1$ causes the reappearance of a pupal cuticle gene $E d g 78 E$ and suppresses a larval cuticle gene $L c p 65 A-b$. Z3 and Z4 both suppress the larval cuticle gene, while Z2 activates the pupal cuticle gene. During the adult molt, misexpression of $\mathrm{Zl}$ activates the pupal cuticle gene and inhibits an adult cuticle gene Acp65A. These data indicate that different isoforms have varying effects on these cuticle genes. It also has been shown in Drosophila melanogaster that different BR isoforms can have opposite regulatory functions on the same gene. For example, in regulating the $D d c$ gene at the end of the molt, Z2 functions as an activator, while either Z1 or Z4 acts as a repressor (Andres et al. 1993, Hodgetts et al. 1995).

Effects of mosquito BR isoforms on $V g$ gene expression have been tested by the cell transfection analysis. This analysis confirmed the ability of BR isoforms to alter transactivation of the $V g$ gene. Significantly, all BR isoforms have no functions by themselves on the $V g$ promoter. However, they can enhance or attenuate AaEcR/AaUSP-mediated 20E activation of the $V g$ promoter. $\mathrm{Z} 1$ and $\mathrm{Z} 4$ have very similar protein structure and expression profiles during vitellogenesis and both of them repressed AaEcR/AaUSP-mediated activation of the $V g$ promoter in the cell transfection assay. In contrast, Z2 enhanced AaEcR/AaUSP-mediated activation of the $V g$ promoter. Z3 had no obvious effect on the $V g$ promoter in cell transfection assay. Together, these results suggest that the BR isoforms are essential for proper activation and termination of the $V g$ gene in response to $20 \mathrm{E}$.

\section{Acknowledgements}

This work was supported by a grant AI-36959 from the National Institutes of Health to A S R. We 
thank Mr Andrew Hufford for editing the manuscript.

\section{References}

Ahmad KF, Engel GK \& Prive GG 1998 Crystal structure of the BTB domain from PLZF. PNAS 95 12123-12128.

Andres AJ, Fletcher JC, Karim FD \& Thummel CS 1993 Molecular analysis of the initiation of insect metamorphosis: a comparative study of Drosophila ecdysteroid-regulated transcription. Developmental Biology 160 388-404.

Ashburner M 1972 Patterns of puffing activity in the salivary gland chromosomes of Drosophila. VI. Induction by ecdysone in salivary glands of D. melanogaster cultured in vitro. Chromosoma 38 255-281.

Bardwell VJ \& Treisman R 1994 The POZ domain: a conserved protein-protein interaction motif. Genes and Development $\mathbf{8}$ 1664-1677.

Bayer CA, Holley B \& Fristrom JW 1996 A switch in broad-complex zinc-finger isoform expression is regulated posttranscriptionally during the metamorphosis of Drosophila imaginal discs. Developmental Biology 177 1-14.

Bayer CA, von Kalm L \& Fristrom JW 1997 Relationships between protein isoforms and genetic functions demonstrate functional redundancy at the Broad-Complex during Drosophila metamorphosis. Developmental Biology 187 267-282.

Baver C, Zhou X, Zhou B, Riddiford LM \& von Kalm L 2003 Evolution of the Drosophila broad locus: the Manduca sexta broad Z4 isoform has biological activity in Drosophila. Development Genes and Evolution $213471-476$.

Belyaeva ES, Aizenzon MG, Semeshin VF, Kiss II, Koczka K, Baritcheva EM, Gorelova TD \& Zhimulev IF 1980 Cytogenetic analysis of the 2B3-4-2B11 region of the $\mathrm{X}$-chromosome of Drosophila melanogaster. I. Cytology of the region and mutant complementation groups. Chromosoma 81 281-306.

Belyaeva ES, Vlassova IE, Biyasheva ZM, Kakpakov VT, Richards G \& Zhimulev IF 1981 Cytogenetic analysis of the 2B3-4-2B11 region of the $\mathrm{X}$ chromosome of Drosophila melanogaster. II. Changes in 20-OH ecdysone puffing caused by genetic defects of puff 2B5. Chromosoma 84 207-219.

Burtis KC, Thummel CS, Jones CW, Karim FD \& Hogness DS 1990 The Drosophila 74Ef early puff contains E74, a complex ecdysone-inducible gene that encodes 2 Ets-related proteins. Cell 61 85-99.

Cherbas L, Lee K \& Cherbas P 1991 Identification of ecdysone response elements by analysis of the Drosophila Eip28/29 gene. Genes and Development 5 120-131.

Cho WL, Deitsch KW \& Raikhel AS 1991 An extraovarian protein accumulated in mosquito oocytes is a carboxypeptidase activated in embryos. PNAS 88 10821-10824.

Cho WL \& Raikhel AS 1992 Cloning of cDNA for mosquito lysosomal aspartic protease-sequence - analysis of an insect lysosomal-enzyme similar to cathepsin-D and cathepsin-E. Fournal of Biological Chemistry 267 21823-21829.

Cho WL, Tsao SM, Hays AR, Walter R, Chen JS, Snigirevskaya ES \& Raikhel AS 1999 Mosquito cathepsin B-like protease involved in embryonic degradation of vitellin is produced as a latent extraovarian precursor. Fournal of Biological Chemistry $\mathbf{2 7 4}$ $13311-13321$

Crossgrove K, Bayer CA, Fristrom JW \& Guild GM 1996 The Drosophila Broad-Complex early gene directly regulates late gene transcription during the ecdysone-induced puffing cascade. Developmental Biology 180 745-758.

Deng WM \& Bownes M 1997 Two signaling pathways specify localized expression of the Broad-Complex in Drosophila eggshell patterning and morphogenesis. Development 124 $4639-4647$.
Deitsch KW, Chen JS \& Raikhel AS 1995 Indirect control of yolk protein genes by 20-hydroxyecdysone in the fat-body of the mosquito, Aedes aegypti. Insect Biochemistry and Molecular Biology 25 449-454.

Dhadialla TS \& Raikhel AS 1994 Endocrinology of mosquito vitellogenesis. In Perspectives in Comparative Endocrinology, pp 275-281. Eds KG Davey, RE Peter \& SS Tobe. Ottawa, Canada: National Research Council of Canada.

DiBello PR, Withers DA, Bayer CA, Fristrom JW \& Guild GM 1991 The Drosophila Broad-Complex encodes a family of related proteins containing zinc fingers. Genetics 129 385-397.

Dittmer NT, Sun G, Wang S \& Raikhel AS 2003. Mosquito CREB is involved in the termination of vitellogenesis. Molecular and Cellular Endocrinology 210 39-49.

Flanagan TR \& Hagedorn HH 1977 Vitellogenin synthesis in mosquito-role of juvenile hormone in development of responsiveness to ecdysone. Physiological Entomology 2 173-178.

Fletcher JC \& Thummel CS 1995a The Drosophila E74 gene is required for the proper stage-specific and tissue-specific transcription of ecdysone-regulated genes at the onset of metamorphosis. Development 121 1411-1421.

Fletcher JC \& Thummel CS $1995 b$ The ecdysone-inducible Broad-complex and E74 early genes interact to regulate target gene transcription and Drosophila metamorphosis. Genetics 141 $1025-1035$

Guay PS \& Guild GM 1991 The ecdysone-induced puffing cascade in Drosophila salivary glands: a Broad-Complex early gene regulates intermolt and late gene transcription. Genetics $\mathbf{1 2 9}$ $169-175$.

Hagedorn HH 1983 The role of ecdysteroids in the adult insect. In Endocrinology of Insects, pp 271-304. Eds RGH Downer \& H Laufer. New York: Wiley-Liss Inc.

Hagedorn HH 1985 The role of ecdysteroids in reproduction. In Comprehensive Insect Physiology, Biochemistry and Pharmacology, vol. 8, pp 205-261. Eds GA Kerkut \& LI Gilbert. New York: Pergamon Press.

Hagedorn HH 1989 Physiological roles of hemolymph ecdysteroids in adult insect. In Ecdysone, From Chemistry to Mode of Action, pp 279-289. Ed J Koolman. Stuttgart: Georg Thieme Verlag.

Hagedorn HH, O'Connor JD, Fuchs MS, Sage B, Schlaeger DA \& Bohm MK 1975 Ovary as a source of alpha-ecdysone in an adult mosquito. PNAS 72 3255-3259.

Hagedorn HH, Turner S, Hagedorn EA, Pontecorvo D, Greenbaum P, Pfeiffer D, Wheelock G \& Flanagan TR 1977 Postemergence growth of ovarian follicles of Aedes aegypti. Fournal of Insect Physiology 23 203-206.

Hays AR \& Raikhel AS 1990 A novel protein produced by the vitellogenic fat-body and accumulated in mosquito oocytes. Roux's Archives of Developmental Biology 199 114-121.

Hodgetts RB, Clark WC, O'Keefe SL, Schouls M, Crossgrove K, Guild GM \& von Kalm L 1995 Hormonal induction of Dopa decarboxylase in the epidermis of Drosophila is mediated by the Broad-Complex. Development 121 3913-3922.

$\mathrm{Hu}$ X, Cherbas L \& Cherbas P 2003 Transcription activation by the ecdysone receptor (EcR/USP): identification of activation functions. Molecular Endocrinology 17 716-731.

von Kalm L, Crossgrove K, Von Seggern D, Guild GM \& Beckendorf SK 1994 The Broad-Complex directly controls a tissue-specific response to the steroid hormone ecdysone at the onset of Drosophila metamorphosis. EMBO fournal 13 3505-3516.

Karim FD, Guild GM \& Thummel CS 1993 The Drosophila Broad-Complex plays a key role in controlling ecdysone-regulated gene expression at the onset of metamorphosis. Development 118 977-988.

Kiss I, Szabad J \& Major J 1978 Genetic and developmental analysis of puparium formation in Drosophila. Molecular and General Genetics $16477-83$ 
Kiss I, Beaton AH, Tardiff J, Fristrom D \& Fristrom JW 1988 Interactions and developmental effects of mutations in the Broad-Complex of Drosophila melanogaster. Genetics 118 247-259.

Koelle MR, Talbot WS, Segraves WA, Bender MT, Cherbas P \& Hogness DS 1991 The Drosophila Ecr gene encodes an ecdysone receptor, a new member of the steroid-receptor superfamily. Cell 67 59-77.

Kokoza VA, Martin D, Mienaltowski MJ, Ahmed A, Morton CM \& Raikhel AS 2001 Transcriptional regulation of the mosquito vitellogenin gene via a blood meal-triggered cascade. Gene $\mathbf{2 7 4}$ $47-65$.

Lea AO 1964 Studies on the dietary and endocrine regulation of autogenous reproduction in Aedes taeniorhynchus (WIED). Fournal of Medical Entomology 39 40-44.

Lepesant J-A, Maschat F, Kejzlarova-Lepesant J, Benes H \& Yanicostas C 1986 Developmental and ecdysteroid regulation of gene expression in the larval fat body of Drosophila melanogaster. Archives of Insect Biochemistry and Physiology Suppl 1 133-141.

Martin D, Wang SF \& Raikhel AS 2001 The vitellogenin gene of the mosquito Aedes aegypti is a direct target of ecdysteroid receptor. Molecular and Cellular Endocrinology 173 75-86.

Mugat B, Brodu V, Kejzlarova-Lepesant J, Antoniewski C, Bayer CA, Fristrom JW \& Lepesant JA 2000 Dynamic expression of broad-complex isoforms mediates temporal control of an ecdysteroid target gene at the onset of Drosophila metamorphosis. Developmental Biology 227 104-117.

Nelson RJ, Odell GM, Christiansen AE \& Laird CD 1991 Hormonal control of gene expression: interactions between two trans-acting regulators in Drosophila. Developmental Biology 144 $152-166$.

Pierceall WE, Li C, Biran A, Miura K, Raikhel AS \& Segraves WA 1999 E75 expression in mosquito ovary and fat body suggests reiterative use of ecdysone-regulated hierarchies in development and reproduction. Molecular and Cellular Endocrinology 150 73-89.

Raikhel AS 1992 Vitellogenesis in mosquitoes. Advances in Disease Vector Research 9 1-39.

Raikhel AS, McGurk L \& Bownes M 2003 Ecdysteroid action insect reproduction. In Encyclopedia of Hormones, pp 451-459. Eds HL Henry \& AW Norman. San Diego; Academic Press.

Restifo LL \& Merrill VK 1994 Two Drosophila regulatory genes, deformed and the Broad-Complex, share common functions in development of adult GNS, head, and salivary glands. Developmental Biology 162 465-485.

Riddiford LM, Curtis AT \& Kiguchi K 1979 Culture of the epidermis of the tobacco hornworm Manduca sexta. Tissue Culture Association Manual 5 975-985.

Segraves WA \& Hogness DS 1990 The E75 ecdysone-inducible gene responsible for the 75B early puff in Drosophila encodes 2 new members of the steroid-receptor superfamily. Genes and Development $4204-219$.

Shapiro JP \& Hagedorn HH 1982 Juvenile hormone and the development of ovarian responsiveness to a brain hormone in the mosquito, Aedes aegypti. General and Comparative Endocrinology $\mathbf{4 6}$ $176-183$.

Stewart M, Murphy C \& Fristrom JW 1972 The recovery and preliminary characterization of X-chromosome mutants affecting imaginal discs of Drosophila melanogaster. Developmental Biology 27 $71-83$.

Sun GQ, Zhu JS, Li C, Tu ZJ \& Raikhel AS 2002 Two isoforms of the early E74 gene, an Ets transcription factor homologue, are implicated in the ecdysteroid hierarchy governing vitellogenesis of the mosquito, Aedes aegypti. Molecular and Cellular Endocrinology 190 $147-157$
Sun GQ Zhu JS \& Raikhel AS 2004 The early gene E74B isoform is a transcriptional activator of the ecdysteroid regulatory hierarchy in mosquito vitellogenesis. Molecular and Cellular Endocrinology 218 95-105.

Tzolovsky G, Deng WM, Schlitt T \& Bownes M 1999 The function of the Broad-Complex during Drosophila melanogaster oogenesis. Genetics 153 1371-1383.

Uhlirova M, Foy BD, Beaty BJ, Olson KE, Riddiford LM \& Jindra M 2003 Use of Sindbis virus-mediated RNA interference to demonstrate a conserved role of Broad-Complex in insect metamorphosis. PNAS 100 15607-15612.

Urness LD \& Thummel CS 1995 Molecular analysis of a steroid-induced regulatory hierarchy: the Drosophila E74A protein directly regulates L71-6 transcription. EMBO Fournal 14 6239-6246.

Wang SF, Miura K, Miksicek RJ, Segraves WA \& Raikhel AS 1998 DNA binding and transactivation characteristics of the mosquito ecdysone receptor-Ultraspiracle complex. Fournal of Biological Chemistry 273 27531-27540.

Wang SF, Li C, Zhu JS, Miura K, Miksicek RJ \& Raikhel AS 2000 Differential expression and regulation by 20-hydroxyecdysone of mosquito ultraspiracle isoforms. Developmental Biology 218 99-113.

Wang SF, Li C, Zhu JS \& Raikhel AS 2002 Differential expression and regulation of mosquito ecdysteroid receptor isoforms by 20-hydroxyecdysone. Molecular and Cellular Endocrinology 196 $29-42$.

Yao TP, Segraves WA, Oro AE, McKeown M \& Evans RM 1992 Drosophila ultraspiracle modulates ecdysone receptor function via heterodimer formation. Cell 71 63-72.

Yao TP, Forman BM, Jiang ZY, Gherbas L, Chen JD, McKeown M, Cherbas P \& Evans RM 1993 Functional ecdysone receptor is the product of Ecr and ultraspiracle genes. Nature 366 476-479.

Zhou BH \& Riddiford LM 2001 Hormonal regulation and patterning of the Broad-Complex in the epidermis and wing discs of the tobacco hornworm, Manduca sexta. Developmental Biology 231 $125-137$.

Zhou X \& Riddiford LM 2002 Broad specifies pupal development and mediates the 'status quo' action of juvenile hormone on the pupal-adult transformation in Drosophila and Manduca. Development 129 2259-2269.

Zhou B, Hiruma K, Shinoda T \& Riddiford LM 1998 Juvenile hormone prevents ecdysteroid-induced expression of broad complex RNAs in the epidermis of the tobacco hornworm, Manduca sexta. Developmental Biology 203 233-244.

Zhu J, Miura K, Chen L \& Raikhel AS $2003 a$ Cyclicity of mosquito vitellogenic ecdysteroid-mediated signaling is modulated by alternative dimerization of the RXR homologue Ultraspiracle. PNAS 100 544-549.

Zhu J, Chen L \& Raikhel AS 2003b Posttranscriptional control of the competence factor betaFTZ-F1 by juvenile hormone in the mosquito Aedes aegypti. PNAS 100 13338-13343.

Zollman S, Godt D, Prive GG, Couderc JL \& Laski FA 1994 The BTB domain, found primarily in zinc finger proteins, defines an evolutionarily conserved family that includes several developmentally regulated genes in Drosophila. PNAS 91 10717-10721.

Received 31 August 2004

Accepted 14 September 2004

Made available online as an

Accepted Preprint 27 September 2004 